\title{
Renormalized One-loop Theory of Correlations in Polymer Blends
}

\author{
Jian Qin and David C. Morse \\ Department of Chemical Engineering and Materials Science, \\ University of Minnesota, 421 Washington Ave. S.E., Minneapolis, MN 55455
}

(Dated: March 14, 2021)

\begin{abstract}
The renormalized one-loop theory is a coarse-grained theory of corrections to the self-consistent field theory (SCFT) of polymer liquids, and to the random phase approximation (RPA) theory of composition fluctuations. We present predictions of corrections to the RPA for the structure function $S(k)$ and to the random walk model of single-chain statics in binary homopolymer blends. We consider an apparent interaction parameter $\chi_{a}$ that is defined by applying the RPA to the small $k$ limit of $S(k)$. The predicted deviation of $\chi_{a}$ from its long chain limit is proportional to $N^{-1 / 2}$, where $N$ is chain length. This deviation is positive (i.e., destabilizing) for weakly non-ideal mixtures, with $\chi_{a} N \lesssim 1$, but negative (stabilizing) near the critical point. The positive correction to $\chi_{a}$ for low values of $\chi_{a} N$ is a result of the fact that monomers in mixtures of shorter chains are slightly less strongly shielded from intermolecular contacts. The depression in $\chi_{a}$ near the critical point is a result of long-wavelength composition fluctuations. The one-loop theory predicts a shift in the critical temperature of $\mathcal{O}\left(N^{-1 / 2}\right)$, which is much greater than the predicted $\mathcal{O}\left(N^{-1}\right)$ width of the Ginzburg region. Chain dimensions deviate slightly from those of a random walk even in a one-component melt, and contract slightly with increasing $\chi_{e}$. Predictions for $S(k)$ and single-chain properties are compared to published lattice Monte Carlo simulations.
\end{abstract}

\section{INTRODUCTION}

Equilibrium properties of polymer mixtures that involve correlations over distances greater than a few Angstroms are rather well described by a family of closely related selfconsistent field theories. Among these are Flory-Huggins (FH) theory for the free energy of a homogeneous mixture and the random phase approximation (RPA) theory of composition fluctuations. Both of these are special cases of a more general self-consistent field theory (SCFT) of inhomogenous polymer liquids.

Theoretical treatments of polymer mixtures that go beyond SCFT may be roughly classified as either microscopic liquid state theories or coarse-grained theories of correlation effects. Liquid state theories such as the polymer reference interaction site model (PRISM) $\stackrel{1}{\underline{1}}$ and Born-GreenYvon theories ${ }^{2}$ are designed to treat models with strong repulsive interactions, and attempt to predict all details of liquid structure from first principles. Coarse-grained theories are based on more idealized models of chain conformations and interactions, e.g., as continuous Gaussian chains with point-like interactions, similar to those used in coarsegrained SCFT.

Over the past 20 years, a series of coarse-grained theories of correlation effects in polymer mixtures have made use of a functional integral representation of the partition function that was originally introduced by Edwards ${ }^{3,4.5,6,7.8 .9}$ All of these "field theoretic" studies of mixtures have thus far used an approximation in which the distribution of field fluctuations is approximated by a Gaussian functional. This approximation is referred to in the jargon of field theory (which we have adopted) as a "oneloop" approximation. An alternative way of deriving this theory, which we find more informative, is discussed in Sec. (VI) of this paper.

\section{A. Coarse-Graining and Renormalization}

The reason for studying correlations in coarse-grained models is the hope that, by doing so, one might describe phenomena that are not captured by SCFT, but that are, in some sense, independent of short-wavelength details. The first several studies of coarse-grained models of blends $s^{3,45,6,7.8}$ were, however, plagued by a sensitivity of all predictions to the treatment of very short wavelength correlations, at length scales which the coarse-grained Hamiltonian was never intended to describe. The way around this problem, which was first applied in this context by Wang $\stackrel{9}{\text {, }}$ is a renormalization procedure in which the sensitivity to monomer scale structure is absorbed into the values of a few phenomenological parameters. Wang 9 considered the one-loop prediction for the free energy of a homogeneous polymer blend, and showed that the sensitivity of this quantity to local fluid structure could be absorbed into an appropriate renormalization of the Flory-Huggins interaction parameter. More recently, we ${ }^{10}$ showed how a generalization of this procedure can be used to obtain unambiguous results for one-loop corrections to both collective and single-chain correlation functions at nonzero wavenumbers, using the same formalism for polymer blends and diblock copolymer melts.

Development of this renormalization procedure required two insights that were absent from the interpretation of earlier studies of the one-loop theory:

The first was that the SCF approximation should be identified with the infinite chain limit of the one-loop theory, rather than a simple random-mixing approximation. Prior to Wang's work, the SCF approximation had often been conceptually identified with a simple mean-field approximation that is obtained from a saddle-point approximation for the underlying functional integral. This mean-field theory ignores all correlations except those arising from in- 
tramolecular connectivity, which is never a quantitatively useful approximation. Defining SCF parameters by a process of extrapolation to the infinite chain limit instead takes advantage of the fact that local correlations in liquids of long polymers depend only weakly on chain length, even in strongly correlated liquids. The difference between the free energy of a system of finite chains and that of a hypothetical reference system of infinite chains is largely the result of slight differences in long-wavelength correlations. These differences may be adequately described by a coarsegrained model. By treating the interaction free energy of an infinite-chain reference system as an input to the theory (which must be inferred from experimental or simulation data), one can obtain a renormalized theory that makes unambiguous predictions of corrections to SCF phenomenology.

A second necessary insight was that the one-loop prediction for the structure factor $S(k)$ at nonzero wavenumber $k$ is affected by a renormalization of statistical segment length, as well as of the interaction free energy. The value of the statistical segment length $b$ of a flexible polymer in a dense mixture is generally different than the "bare" value required as an input to the theory. The one-loop predictions for the magnitude of this difference ${ }^{8}$, are sensitive to short-wavelength correlations. This renormalization of $b$ has no effect upon the predicted value of structure factor $S(k)$ at exactly $k=0$, and so did not need to be taken into account in Wang's interpretation of one-loop predictions for $S(k=0)$. It becomes an unavoidable complication, however, in calculations of single-chain correlations, and in applications to diblock copolymer melts, for which the dominant fluctuations occur at nonzero $k$.

In our previous paper ${ }^{10}$, we focused primarily on clarifying the physical reasoning underlying our renormalization procedure, and on demonstrating that it yields a physically consistent interpretation. In this paper, we present predictions for binary homopolymer blends. Predictions for diblock copolymer melts will be presented elsewhere.

\section{B. Related Prior Work}

The analysis given here has several particularly close antecedents.

Our analysis of the free-energy density of a homogeneous blend and the long wavelength limit of $S(k=0)$, which is given in Secs. II and III is closely related to that of Wang. 9 The main differences arise from mathematical approximations that Wang introduced in order to obtain a completely analytic approximation for behavior near the spinodal. We have avoided all approximations other than the one-loop approximation itself, at the cost of some additional numerical analysis. The additional rigor has allowed us to calculate some additional quantities (e.g., the absolute shift in the critical point) and to identify physical phenomena that are important far from the spinodal.
Our analysis of corrections to Gaussian chain statistics in blends, given in Sec. IV is closely related to the recent theoretical analysis of chain statistics in one-component melts by Beckrich, Johner, Semenov, and coworkers ${ }^{11,12}$. Like us, but prior to our work, these authors introduced a renormalized statistical segment length, defined by extrapolation to $N=\infty$, as part of a systematic calculation of small corrections to random-walk statistics. We initially failed to notice the connection to our own work $\stackrel{10}{=}$ or to cite their work. These authors presented a beautiful analysis of universal corrections to Gaussian statistics in an incompressible melt at intermediate wavelengths, less than the coil size but greater than a monomer size. They also obtained analytic results for one-loop corrections to the intramolecular correlation function at all wavenumbers in melts of equilibrium polymers, with an exponential distribution of chain lengths. By introducing a numerical procedure for extracting UV convergent parts of divergent integrals, we are also able to numerically evaluate one-loop predictions for melts and blends of monodisperse homopolymers and (elsewhere) for block copolymer melts. Here, we consider statistics of monodisperse polymers in binary blends.

The most important antecedent of both our work and the recent work of the Strasbourg group is the much larger, and (generally) more sophisticated theoretical literature on correlations in semi-dilute polymer solutions. ${ }^{13}$ At a technical level, the closest analog of our approach within this literature seems to be the analysis of mono-disperse semi-dilute solutions by Ohta and Nakanishi. ${ }^{14,15}$

\section{Outline}

The paper is organized as follows: Sec. II provides a brief review of the renormalized one-loop theory for the free energy density, and of our notation. Sec. III presents predictions for corrections to the RPA theory of composition fluctuations. Sec. IV presents predictions for corrections to random walk statistics in polymer blends. Sec. V presents comparisons of theoretical predictions to results of published lattice Monte Carlo simulations. Sec. VI discusses the physical relationship between the one-loop theory for the free energy and RPA theory of correlations. Sec. VII clarifies the physical origin of some features of the oneloop theory by relating them to corresponding features of the underlying RPA theory.

\section{THEORETICAL FORMULATION}

\section{A. Model and Notation}

We consider a coarse-grained model of a binary homopolymer blend. Let $v$ be the average volume per monomer, for either species. Let the volume fraction, degree of polymerization, statistical segment length and pack- 
ing length ${ }^{16}$ for species $i$ be denoted by $\phi_{i}, N_{i}, b_{i}$, and $l_{i} \equiv v / b_{i}^{2}$, respectively, for species $i=1$ and 2 .

Let the potential energy be the sum of an intramolecular potential $U_{\text {chain }}$ and a monomer-monomer pair interaction

$$
U_{\mathrm{int}}=\frac{1}{2} \int d \mathbf{r} \int d \mathbf{r}^{\prime} U_{i j}\left(\mathbf{r}-\mathbf{r}^{\prime}\right) c_{i}(\mathbf{r}) c_{j}\left(\mathbf{r}^{\prime}\right) .
$$

Here, $c_{i}(\mathbf{r})=\sum_{m s}^{i} \delta\left(\mathbf{r}-\mathbf{R}_{m i}(s)\right)$ is the concentration of monomers of type $i$, in which $\mathbf{R}_{m i}(s)$ is the position of monomer $s$ on molecule $m$ of species $i$. The strength of the pair interaction is taken to be of the form

$$
U_{i j}\left(\mathbf{r}-\mathbf{r}^{\prime}\right)=v\left(\begin{array}{cc}
B & B+\chi_{0} \\
B+\chi_{0} & B
\end{array}\right) \delta_{\Lambda}\left(\mathbf{r}-\mathbf{r}^{\prime}\right) .
$$

Here, $\delta_{\Lambda}$ is a function $\delta_{\Lambda} \equiv F\left(\Lambda\left|\mathbf{r}-\mathbf{r}^{\prime}\right|\right) \Lambda^{-3}$ with a range $\Lambda^{-1}$ and an integral $\int d \mathbf{r} u_{\Lambda}=1$, so that $\delta_{\Lambda}$ approaches a Dirac $\delta$-function as $\Lambda \rightarrow \infty$. Here, $B$ is a dimensionless compression energy and $\chi_{0}$ is a dimensionless "bare" interaction parameter. The incompressible limit may obtained by considering the behavior when $B$ is very large.

Let

$$
S_{i j}(\mathbf{r})=\left\langle\delta c_{i}(\mathbf{r}) \delta c_{j}(0)\right\rangle
$$

where $\delta c_{i}(\mathbf{r})=c_{i}(\mathbf{r})-\left\langle c_{i}\right\rangle$, and let $S_{i j}(\mathbf{k})=$ $\int d \mathbf{r} e^{i \mathbf{k} \cdot \mathbf{r}} S_{i j}(\mathbf{r})$ denote the Fourier transform. Let $\Omega_{i}(\mathbf{k})$ be the intramolecular contribution to $S_{i i}(\mathbf{k})$ from chains of type $i$. A continuous random walk model yields

$$
\Omega_{i}(\mathbf{k})=\phi_{i} N g\left(k^{2} R_{g, i}^{2}\right) / v
$$

where $R_{g, i}^{2}=N_{i} b_{i}^{2} / 6$ is the radius of gyration for species $i$ and $g(x)=2\left(e^{-x}-1+x\right) / x^{2}$ is the Debye function.

Composition fluctuations in an effectively incompressible blend may be described by a scalar correlation function

$$
S(k)=S_{11}(k)=S_{22}(k)=-S_{12}(k) \quad .
$$

Here, we follow Schweizer and Curro and our previous work, by expressing this scalar correlation function in terms of what we (and Wang ${ }^{9}$ ) refer to as an "apparent" interaction parameter $\chi_{a}(k)$, by defining

$$
S^{-1}(k)=\Omega_{1}^{-1}(k)+\Omega_{2}^{-1}(k)-2 v \chi_{a}(k) .
$$

This is, of course, a generalization of the RPA expression for $S(k)$, in which it is assumed that chains are random walks and that $\chi_{a}(k)$ is independent of $k, N$, and $\chi N$.

\section{B. One-loop Free Energy}

The Helmholtz free energy $f$ per volume for this coarsegrained model may be expressed, in the incompressible limit, as a sum

$$
f=f_{\text {id }}+e_{\mathrm{mf}}+f_{\text {corr }},
$$

where

$$
f_{\text {id }} \equiv \frac{\phi_{1}}{N_{1} v} \ln \phi_{1}+\frac{\phi_{2}}{N_{2} v} \ln \phi_{2}
$$

is the ideal Flory-Huggins entropy of mixing, and

$$
\begin{aligned}
e_{\mathrm{mf}} & \equiv \frac{1}{2} \int d \mathbf{r} U_{i j}(\mathbf{r})\left\langle c_{i}\right\rangle\left\langle c_{j}\right\rangle \\
& =v^{-1} \chi_{0} \phi_{1} \phi_{2}
\end{aligned}
$$

is a simple mean-field approximation for the interaction energy density. The remainder, denoted $f_{\text {corr }}$, is a correlation free energy. Here, and throughout the remainder of this paper except Sec. V, where we compare to simulation results, we set $k T=1$.

A one-loop approximation for $f_{\text {corr }}$ has been obtained in previous work by considering a Gaussian approximation for the distribution of fluctuations of a complex chemical potential field within the context of the Edwards auxiliary field formalism. In Sec. VI we discuss an alternative derivation in which this approximation is instead obtained by thermodynamic integration of the RPA correlation function. However it is obtained, the resulting approximation for $f_{\text {corr }}$ is given, in the general case of a compressible liquid, by a Fourier integral

$$
f_{\text {corr }}=\frac{1}{2} \int_{\mathbf{q}} \ln [\operatorname{det}|\mathbf{I}+\boldsymbol{\Omega}(\mathbf{q}) \mathbf{U}(\mathbf{q})|]
$$

Here, the bold faced symbols $\boldsymbol{\Omega}(\mathbf{q})$ and $\mathbf{U}(\mathbf{k})$ indicate $2 \times 2$ matrices with elements $\delta_{i j} \Omega_{i}(\mathbf{q})$ and $U_{i j}(\mathbf{q})$, respectively, I denotes the identity, and $\operatorname{det}|\cdots|$ is a determinant. Here and hereafter, we use the shorthand $\int_{\mathbf{q}} \equiv \int d^{3} q /(2 \pi)^{3}$ for Fourier integrals.

In the nearly-incompressible limit of interest here, Eq. 10 for $f_{\text {corr }}$ may be approximately reduced to

$$
f_{\text {corr }}=\frac{1}{2} \int_{\mathbf{q}} \ln \left[\left(\Omega_{1}+\Omega_{2}-2 \chi_{0} v \Omega_{1} \Omega_{2}\right) v\right] .
$$

To obtain the integrand of Eq. (11), in a model with a pair interaction of the form given in Eq. (2), we have both taken the limit $B \gg 1$ in Eq. (10), and also replaced the Fourier transform of $\delta_{\Lambda}$ by unity. This yields an expression for the integrand that is valid for $|\mathbf{q}| \ll \Lambda$. In what follows, we regularize the one-loop approximation for $f_{\text {corr }}$ by treating $\Lambda$ as a cutoff wavenumber, and simply restricting the integral in Eq. (11) to $|\mathbf{q}|<\Lambda$. This is equivalent to the use of a pair potential in which the Fourier transform of $\delta_{\Lambda}\left(\mathbf{r}-\mathbf{r}^{\prime}\right)$ is taken to be unity for $\mathbf{q}<\Lambda$, and zero for $\mathbf{q}>\Lambda$. The purpose of the renormalization procedure discussed below is to extract predictions that are independent of such details of the treatment of short-wavelength correlations.

\section{Divergence and Renormalization}

It has long been known that, in a model of continuous Gaussian chains, the idealized limit $\Lambda \rightarrow \infty$ of point-like 
interactions yields a one-loop expression for $f_{\text {corr }}$ that diverges as the cube of $\Lambda .3,4,5,9$, In previous work, both Wang 9 and we ${ }^{10}$ have analyzed the structure of the high- $q$ divergence of this free energy density, and its physical significance. We found that Eq. (11) for $f_{\text {corr }}$ can be expressed as a sum

$$
f_{\text {corr }}=f_{\text {corr }}^{\Lambda}+f^{*}
$$

of an "ultra-violet" (UV) divergent contribution $f_{\text {corr }}^{\Lambda}$, which increases with increasing $\Lambda$, and a contribution $f^{*}$ that is independent of $\Lambda$. The UV divergent contribution $f_{\text {corr }}^{\Lambda}$ may be expressed as a sum ${ }^{10}$

$$
f_{\mathrm{corr}}^{\Lambda}=f_{\mathrm{bulk}}^{\Lambda}+f_{\mathrm{end}}^{\Lambda}
$$

where

$$
\begin{aligned}
f_{\text {bulk }}^{\Lambda} & =\frac{1}{12 \pi^{2}}\left[\ln \left(\frac{12 \bar{l}}{v \Lambda^{2}}\right)+\frac{2}{3}\right] \Lambda^{3}-\frac{6 \chi_{0}}{\pi^{2} v} \frac{l_{1} l_{2}}{\bar{l}} \phi_{1} \phi_{2} \Lambda \\
f_{\text {end }}^{\Lambda} & =-\frac{3}{2 \pi^{2} v \bar{l}}\left(\frac{\phi_{1} l_{1}^{2}}{N_{1}}+\frac{\phi_{2} l_{2}^{2}}{N_{2}}\right) \Lambda
\end{aligned}
$$

and where $\bar{l} \equiv \phi_{1} l_{1}+\phi_{2} l_{2}$. The contribution $f_{\text {end }}^{\Lambda}$, which is proportional to $1 / N$, was identified ${ }^{10}$ as an excess free energy for chain ends. The quantity $f_{\text {bulk }}^{\Lambda}$, which is independent of $N$, is instead a correction to the "bulk" interaction free energy density of a hypothetical reference system of infinitely long chains.

To remove the explicit cutoff dependence, we reinterpret the one-loop free energy (excluding end effects) as a sum

$$
f=f_{\text {id }}+f_{\text {int }}+f_{\text {end }}^{\Lambda}+f^{*} .
$$

in which

$$
f_{\text {int }} \equiv e_{\mathrm{mf}}+f_{\mathrm{bulk}}^{\Lambda}
$$

is identified as the interaction free energy of the relevant form of SCFT. The contribution $f_{\text {end }}^{\Lambda}$ is a non-universal chain end contribution that could (and generally should) be added to SCFT, to reflect the fact that the fluid structure is perturbed in the immediate vicinity of any chain end. The remaining cutoff-independent contribution $f^{*}$, is identified as a universal correction to the SCF phenomenology.

Eq. (16) for $f_{\text {int }}$ simplifies considerably in the case of structurally symmetric polymers, with $l_{1}=l_{2}=l$. In this case $f_{\text {int }}$ is given, to within a composition-independent constant, by a function of the form

$$
f_{\text {int }}=v^{-1} \chi_{e}(\Lambda) \phi_{1} \phi_{2}
$$

where

$$
\chi_{e}(\Lambda)=\left[1-\frac{6}{\pi^{2}} l \Lambda\right] \chi_{0} .
$$

is an effective Flory-Huggins interaction parameter. This UV divergent expression for $\chi_{e}$ was first obtained by
Olvera de la Cruz et al. $\stackrel{3}{.}$. The physical origin of the UV divergence of this quantity is discussed in Sec. VII

We focus in the remainder of this paper on the UV convergent correction $f^{*}$. It is convenient to introduce a notation for $f^{*}$ and related quantities, in which we use $\int_{\mathbf{q}}^{*}$ to denote a renormalized Fourier integral. The renormalized Fourier integral of an integrand $A(\mathbf{q})$ is defined by a difference

$$
\int_{\mathbf{q}}^{*} A(\mathbf{q}) \equiv \int_{\mathbf{q}} A(\mathbf{q})-\int_{\mathbf{q}}^{\Lambda} A(\mathbf{q})
$$

between the unrenormalized integral $\int_{\mathbf{q}} A(\mathbf{q})$ and the UV divergent part of the integral, denoted by $\int_{\mathbf{q}}^{\Lambda} A(\mathbf{q})$. In a theory of continuous chains, the "UV divergent" part of Fourier integral is obtained by constructing a high- $q$ asymptotic expansion of the integrand, in decreasing powers of $q R$, and retaining only those terms in the expansion that lead to a UV divergent integral 10

With this notation, we may write

$$
f^{*}=\frac{1}{2} \int_{\mathbf{q}}^{*} \ln \left[\left(\Omega_{1}+\Omega_{2}-2 v \chi \Omega_{1} \Omega_{2}\right) v\right] .
$$

The $N$-dependence of $f^{*}$ may be isolated by nondimensionalizing the renormalized integral in terms of a reference length $R$, which we take to be the root-meansquared end-to-end $R=R_{g 1}$ of species 1 . It may be shown that all of the quantities in the integrand can then be expressed as functions of $q R$ and of a set of dimensionless parameters $\phi_{1}, \chi N, N_{2} / N_{1}, R_{2} / R_{1}$. By this method, we find that $f^{*}$ may be expressed as a function of the form

$$
f^{*}=\frac{1}{R^{3}} \hat{f}^{*}\left(\chi N, \phi_{1}, N_{2} / N_{1}, R_{2} / R_{1}\right)
$$

in which $\hat{f}^{*}$ is a dimensionless function given by the value of the non-dimensionalized renormalized integral. The required set of dimensionless parameters is the same as that required by the standard non-dimensionalized SCFT for an incompressible blend. Note that, in Eqs. (20) and (21), we have written $f_{\text {corr }}$ as a product of a parameter $\chi$, but have not specified whether by $\chi$ we mean $\chi_{0}, \chi_{e}$, or (perhaps) the low- $q$ limit of $\chi_{a}$. In fact, different choices lead to different variants of the theory, as discussed in more detail below.

Results for $f^{*}$ and related quantities that are reported here have all been obtained by numerically evaluating the relevant renormalized Fourier integrals. To do this, for each quantity of interest, we first numerically evaluate the unrenormalized integral with a large but finite cutoff $|\mathbf{q}|<\Lambda$, and then subtract an analytic result for the UV divergent part of the regularized integral. The Fourier integrals required by the one-loop theory may all be expressed as either one dimensional integrals with respect to a wavenumber $q$ (for free energy density and its derivatives) or twodimensional integrals with respect to $q$ and a polar angle 
$\theta$ (for correlation functions at nonzero wavenumber). Subtraction of the UV divergent part from an unrenormalized integral yields an estimate of the renormalized integral that depends only weakly on the numerical cutoff $\Lambda$. Our final result for the value of a renormalized integral is obtained by repeating this procedure for several values of $\Lambda \gg R$, and numerically extrapolating to $\Lambda=\infty$.

\section{Structure Function}

The long-wavelength limit of $S(k)$ is related to the composition dependence of the free energy density $f$ by a statistical mechanical theorem:

$$
\lim _{k \rightarrow 0} S^{-1}(k)=v^{2} \frac{\partial^{2} f}{\partial \phi_{1}^{2}} .
$$

The quantity $S^{-1}(0)$ is also related to the long-wavelength limit of $\chi_{a}(k)$ by

$$
\lim _{k \rightarrow 0} S^{-1}(k)=2 v\left(\chi_{s}-\chi_{a}\right) .
$$

Here, and hereafter, we use $\chi_{a}$ to denote the $\mathbf{q} \rightarrow 0$ limit of the quantity $\chi_{a}(\mathbf{k})$ defined in Eq. (6). The quantity $\chi_{s}$ is the spinodal value of $\chi_{a}$, given by

$$
2 \chi_{s} \equiv v \frac{\partial^{2} f_{\text {id }}}{\partial \phi_{1}^{2}}=\frac{1}{N_{1} \phi_{1}}+\frac{1}{N_{2} \phi_{2}}
$$

Using decomposition (15) for $f$, and hereafter neglecting the explicit end effects, the one-loop approximation for $\chi_{a}$ may be expressed as a sum

$$
\chi_{a}=\chi_{e}+\chi^{*},
$$

in which

$$
\chi_{e} \equiv-\frac{v}{2} \frac{\partial^{2} f_{\text {int }}}{\partial \phi_{1}^{2}}
$$

is the interaction parameter of phenomenological SCFT, and

$$
\chi^{*}=-\frac{v}{2} \frac{\partial^{2} f^{*}}{\partial \phi_{1}^{2}}
$$

is a correction to SCFT arising from long-wavelength fluctuations.

An explicit Fourier integral expression for $\chi^{*}$ may be obtained by differentiating the integrand of Eq. (20) for $f^{*}$ twice with respect to $\phi_{1}$. The resulting approximation for $\chi^{*}$ may be expressed in terms of either Edwards' screened interaction $^{10}$ or in terms of the correlation function

$$
S^{-1}(k ; \chi)=\Omega_{1}^{-1}(k)+\Omega_{2}^{-1}(k)-2 v \chi \quad .
$$

When expressed in terms of $S(k)$, it is found that

$$
\chi^{*}=\frac{v}{4} \int_{\mathbf{q}}^{*}\left[\mu^{2}(q) S^{2}(q ; \chi)-\lambda(q) S(q ; \chi)+\eta\right]
$$

where

$$
\begin{aligned}
\mu(q) & =\frac{1}{\phi_{1} \Omega_{1}(q)}-\frac{1}{\phi_{2} \Omega_{2}(q)} \\
\lambda(q) & =-2\left(\frac{1}{\phi_{1}^{2} \Omega_{1}(q)}+\frac{1}{\phi_{2}^{2} \Omega_{2}(q)}\right) \\
\eta & =1 / \phi_{1}^{2}+1 / \phi_{2}^{2} .
\end{aligned}
$$

The quantities $\mu(0)$ and $\lambda(0)$ are proportional to the third and fourth derivatives of $f_{\text {id }}\left(\phi_{1}\right)$ with respect to $\phi_{1}$, respectively. Since $\mu(q), \lambda(q)$ and $\eta$ are independent of $\chi$, the proximity to the spinodal in this expression is controlled by $S(q)$ alone.

By non-dimensionalizing the integral in Eq. (29), we may show that it yields a correction to $N \chi_{a}$ of the form

$$
N \chi^{*}=\frac{1}{\bar{N}^{1 / 2}} \hat{\chi}^{*}\left(\chi N, \phi_{1}, N_{2} / N_{1}, R_{g 2} / R_{g 1}\right),
$$

in which $\hat{\chi}^{*}$ is a non-dimensional function that is defined by the non-dimensionalized integral. Here,

$$
\bar{N} \equiv N b^{6} / v^{2}
$$

is an invariant degree of polymerization, in which (by convention) $N=N_{1}$ and $b=b_{1}$.

In Eqs. 20, 21, and (31), we have intentionally expressed $f^{*}$ and $\chi^{*}$ as functions of an interaction parameter $\chi$, without specifying whether this input parameter should be taken to be the bare parameter $\chi_{0}$ or some type of a renormalized value. In fact, different variants of the renormalized one-loop theory can be obtained by different choices for the interaction parameter used within these Fourier integrals. If we define $f^{*}$ by simply subtracting the UV divergent part of the one-loop expression for $f_{\text {corr }}$, we obtain a theory that is no longer UV divergent, but that retains an explicit dependence upon the unmeasurable "bare" parameter $\chi_{0}$. Following the logic used to construct renormalized expansions of field theories, however, we may also replace $\chi_{0}$ by a renormalized parameter within each integrand to obtain a theory from which all reference to the bare parameter have been removed. This substitution may be formally justified, within the context of a one-loop approximation, by observing that the difference between approximations obtained by using a bare or renormalized parameter in the one-loop theory differ only at second order in a systematic loop expansion.

In the remainder of this paper, we follow Wang 9 by considering a self-consistent one-loop approximation. In this approximation, also known as a "Hartree" approximation, $\chi$ is replaced within the Fourier integral expression for $\chi^{*}$ by $\chi_{a} \equiv \chi_{a}(\mathbf{q}=0)$. In the resulting theory, $\chi_{a}$ is given by a function of the form

$$
\chi_{a}=\chi_{e}+\chi^{*}\left(\phi_{1}, \chi_{a}\right)
$$

Here, $\chi_{e}$ is related to the SCF (i.e., $N \rightarrow \infty$ ) interaction free energy $f_{\text {int }}(\phi)$ by Eq. (26). For purposes of numerical 
evaluation, it is convenient to rewrite Eq. (33) as an explicit expression $\chi_{e}=\chi_{a}-\chi^{*}\left(\phi_{1}, \chi_{a}\right)$ for $\chi_{e}$ as an explicit function of $\chi_{a}$.

\section{COLLECTIVE FLUCTUATIONS}

\section{A. Symmetric Blends, Critical Composition}

Figs. 1 displays predictions of the renormalized Hartree theory for $\chi_{a}$ in blends of structurally symmetric polymers ( $b=b_{1}=b_{2}$ and $N=N_{1}=N_{2}$ ) at their critical composition $\left(\phi_{1}=1 / 2\right)$. It shows predictions for $\chi_{a} N$ as a function of $\chi_{e} N$ blends with three different chain lengths, corresponding to $\bar{N}=64,128$, and 512. The difference between $\chi_{a} N$ from $\chi_{e} N$ is largest for the shortest chains, and vanishes in the limit $N \rightarrow \infty$. The SCF predicts a critical point for a symmetric $\chi_{e} N=2$. The actual critical point for such a blend is reached when $\chi_{a} N=2$.

Fig. 2] shows the underlying dimensionless function $\hat{\chi}^{*} \equiv\left(\chi_{a} N-\chi_{e} N\right) \sqrt{\bar{N}}$ as a function of $\chi_{a} N$. Note that both $\chi_{a}$ and $\chi^{*}$ vanish in the limit $\chi_{e}=0$ for any structurally symmetric mixture, with $b_{1}=b_{2}$ and $N_{1}=N_{2}$, because this is an exact result for such a ideal mixture, in which the two species are physically indistinguishable.

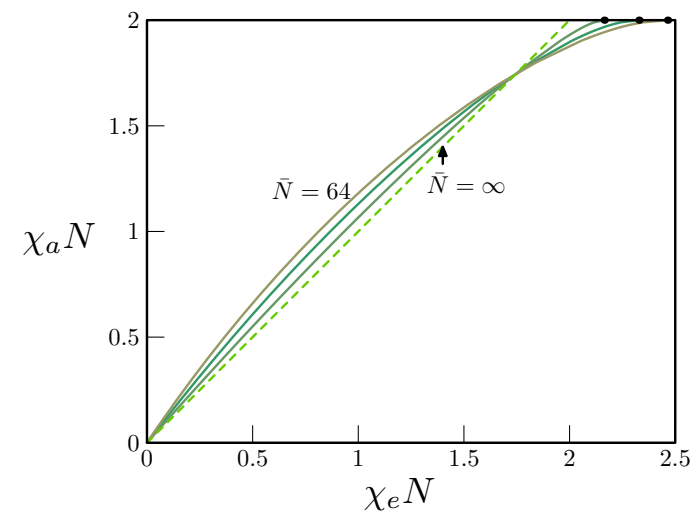

FIG. 1: Self-consistently calculated $\chi_{a} N$ versus $\chi_{e} N$ for symmetric binary blends with $\phi_{1}=1 / 2$ for $\bar{N}=64,128,512$ respectively (solid lines). The dotted line shows where $N \chi_{a}=N \chi_{e}$, which corresponds to the limit $\bar{N} \rightarrow \infty$. Solid dots along the line $\chi_{a} N=2$ indicate critical points for finite chains.

Notably, the dependence of $\chi^{*}$ upon $\chi_{a} N$ is not monotonic: For small values of $\chi_{a} N$, the deviation $\chi^{*}=\chi_{a}-\chi_{e}$ is positive and increases with increasing $\chi_{a}$. Closer to the critical point, $\chi^{*}$ begins to decrease with increasing $\chi_{a} N$, changes sign at $\chi_{a} N \simeq 1.7$, and is negative at the critical point. Note that all the curves in Fig. (1) intersect at $\chi_{a} N=\chi_{e} N \simeq 1.7$, where $\chi^{*}=0$. For any structurally symmetric blend, with $b_{1}=b_{2}$ and $N_{1}=N_{2}$, we find that

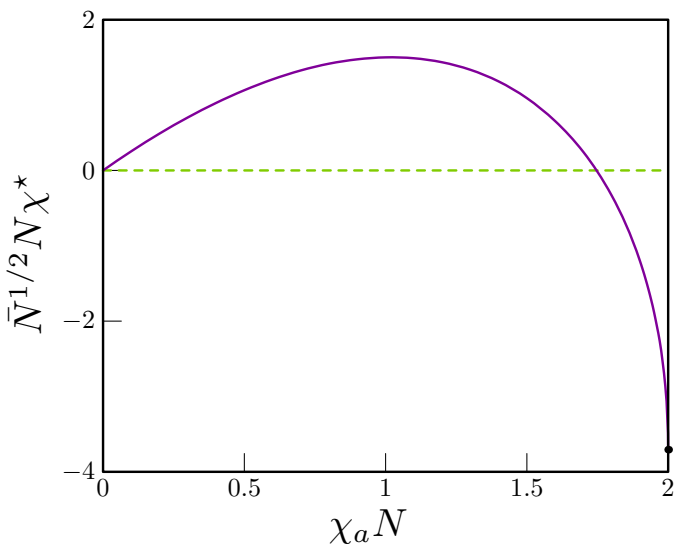

FIG. 2: The deviation $\bar{N}^{1 / 2} N\left(\chi_{a}-\chi_{e}\right)$ as a function of $\chi a N$ for symmetric binary blends at the critical composition, $\phi_{1}=1 / 2$.

the initial slope is

$$
\lim _{\chi_{a} N \rightarrow 0} \frac{\partial\left(\chi^{*} N\right)}{\partial\left(\chi_{a} N\right)}=\frac{(6 / \pi)^{3 / 2}}{\bar{N}^{1 / 2}}
$$

for any composition.

At the critical point of such a blend $\left(\phi_{1}=1 / 2\right.$ and $\chi_{a} N=2$ ), the one-loop prediction for $\chi^{*} N$ approaches a negative limiting value

$$
\lim _{\chi_{a} N \rightarrow 2} \chi^{*} N=-3.7 \bar{N}^{-1 / 2}
$$

with a slope that diverges at the critical point. In the Hartree approximation, this yields a predicted critical value of

$$
\left(\chi_{e} N\right)_{c}=2+3.7 \bar{N}^{-1 / 2} \text {. }
$$

If $\chi_{e}$ is a decreasing function of temperature, this corresponds to a depression in the critical temperature $T_{c}$ by an amount $\delta T_{c} \propto N^{-1 / 2}$

The predicted decrease in $\chi^{*}$ near the critical point is not surprising: Near the critical point, long wavelength correlations decrease the level of local mixing between $A$ and $B$ monomers, thereby decreasing the apparent interaction parameter $\chi_{a}$ and stabilizing the homogeneous phase.

The origin of the positive values obtained for $\chi^{*}$ at small values of $\chi_{a}$ is less obvious. In Sec. VII of this paper we show that this prediction is the result of a subtle dependence of local correlations in a polymer liquid upon overall chain length, which is discussed in much greater detail in an accompanying paper. In a dense liquid of long polymers, the immediate vicinity of each monomer is crowded with monomers that belong to the same chain. This intramolecular "self-concentration" reduces the space available for monomers from other chains, thus creating a so-called correlation hole in the intermolecular correlation function. In a mixture, the correlation hole reduces the magnitude of the potential energy of interaction between monomers of 
types 1 and 2 . We show in the accompanying paper that the depth of the correlation hole, decreases slightly with decreasing chain length. For from the spinodal, this simple packing effect causes $\chi_{a}$ to increase with decreasing chain length, reflecting the fact that shorter chains are less strongly shielded from intermoecular contacts. Because we define $\chi_{e}$ to be the limiting value of $\chi_{a}$ in the limit of infinitely long chains, this effect tends makes a positive contribution to the difference $\chi_{a}(N)-\chi_{e}$ for any finite $N$. In the accompanying paper, we analyze this effect without reference to the one-loop theory, and derive the prefactor in $\mathrm{Eq} 34$ by very different reasoning than that used here.

A shift in the critical temperature by an amount $\delta T_{c} \propto$ $\bar{N}^{-1 / 2}$ was predicted previously by Holyst and Vilgis ${ }^{6}$. The one-loop theory of Holyst and Vilgis did not use a systematic renormalization procedure, but instead introduced an ad hoc cutoff $\Lambda \sim 1 / R$ to avoid the UV divergence. This approach captured the correct scaling, but does not allow a meaningful calculation of numerical values for oneloop corrections, because it yields corrections whose values are sensitive to the exact numerical value chosen for an arbitrary cutoff length.

\section{B. Vicinity of the Spinodal}

We now discuss the behavior of the Hartree theory near the spinodal. This part of our analysis is similar to that given by Wang9 ${ }^{9}$, who focused on behavior near the spinodal, but who made several approximations that we avoid.

A rigorous asymptotic expansion of the behavior of $\chi_{a}$ near the spinodal may be obtained by expanding $S\left(q ; \chi_{a}\right)$, $\mu(q)$ and $\lambda(q)$ in Eq. (29) around their values at $q=0$ and $\chi_{a}=\chi_{s}$. Let

$$
\begin{aligned}
r & =2\left(\chi_{s}-\chi_{a}\right) \\
\tau & =2\left(\chi_{s}-\chi_{e}\right)
\end{aligned}
$$

The required asymptotic expansion of $S(q)$ is

$$
S^{-1}(q) \simeq r+q^{2} \xi_{0}^{2}
$$

where

$$
\xi_{0}^{2} \equiv v \bar{l} /\left(18 \phi_{1} \phi_{2} l_{1} l_{2}\right)
$$

By also expanding $\mu(q)$ and $\lambda(q)$ around $q=0$, we obtain an asymptotic expansion of the form

$$
r=\tau+\frac{v}{\xi_{0}^{3} N^{3 / 2}}\left[\frac{A}{\sqrt{N r}}+B+C \sqrt{N r}+\cdots\right]
$$

where $A, B$, and $C$ are dimensionless coefficients that are independent of $r$, but that that depend upon $\phi_{1}, N_{2} / N_{1}$, and $R_{2} / R_{1}$. Here, $N$ is an arbitrary choice of a reference degree of polymerization, which could be taken to be $N_{1}$ or $N_{2}$ or an appropriate average of the two.
The coefficients $A$ and $C$ in this expansion can be calculated analytically, by considering the singular behavor of the integral near $q=0$ as $r \rightarrow 0$. The coefficient $A$ is given

$$
\begin{aligned}
A\left(\phi_{1}\right) & =\frac{-N^{2}}{16 \pi v^{2}} \mu^{2}(0) \\
& =-\frac{N^{2}}{32 \pi}\left(\frac{1}{\phi_{1}^{2} N_{1}}-\frac{1}{\phi_{2}^{2} N_{2}}\right)^{2} .
\end{aligned}
$$

Here, $\mu(q=0)$ is the third derivative with respect to $\phi_{1}$ of the ideal mixture free energy per monomer $v f_{\text {id }}\left(\phi_{1}\right)$. This quantity vanishes at the Flory-Huggins critical composition, at which $\phi_{1}^{2} N_{1}=\phi_{2}^{2} N_{2}$, and so (as noted by Wang ${ }^{9}$ ) $A\left(\phi_{1}\right)$ also vanishes at the critical composition $\phi_{1 c}$. At the critical composition, we find

$$
C\left(\phi_{1 c}\right)=\frac{-1}{4 \pi \phi_{1 c}^{2} \phi_{2 c}^{2}} \frac{N}{\sqrt{N_{1} N_{2}}} .
$$

These values for $A$ and $C$ agree with corresponding results of Wang 9 .

In the Hartree approximation, the value $\tau_{c}$ of $\tau$ (the reduced "temperature") at the critical point is determined by the value of the constant $B$ at the critical composition: The critical point occurs when $r=0$ at $\phi_{1}=\phi_{1 c}$, or when

$$
\tau_{c}=-v B\left(\phi_{1 c}\right) \xi_{0}^{-3} N^{-3 / 2}
$$

If $B$ is nonzero, an expansion of this form thus yields a shift in the critical value of $\chi_{e} N$ of order $\bar{N}^{-1 / 2}$.

The coefficient $B$ cannot be obtained from an asymptotic analysis of the low- $q$ behavior of the integral in the Eq. 29). To understand why, recall that the problem actually involves three length scales: the monomer size (or cutoff length $\Lambda^{-1}$ ), the coil size $R \propto \sqrt{N}$, and correlation length $\xi=\xi_{0} / \sqrt{r}$. Very near the spinodal, $\xi \gg R$. Our renormalization procedure removes all dependence on $\Lambda^{-1}$, but leaves a dependence on both $R$ and $\xi$. Away from the critical composition, the integral in Eq. (29) develops an infra-red (IR) divergence of the form $A / \sqrt{r}$ as $r \rightarrow 0$. This divergence is the result of fluctuations with wavenumbers $q \sim \xi^{-1}$, and so its prefactor can be obtained by considering the behavior of the integrand near $q=0$. At the critical composition, the coefficient $C$ can be isolated by considering the IR divergence of the derivative of Eq. (29) $\chi^{*}$ with respect to $\chi_{a}$, which then diverges as $r^{-1 / 2}$. The actual value of $\chi^{*}$, however, convergences even at the critical point, and is dominated by fluctuations with $q R \sim 1$. As a result, the one-loop prediction for the shift in the critical point can only be calculated by numerically evaluating the renormalized integral, as done here, without introducing approximations for $\Omega_{1}(q)$ and $\Omega_{2}(q)$ that are valid only for $q R \ll 1$.

At the critical composition, the asymptotic expansion of the Hartree theory may be expressed more compactly as a sum

$$
N r=N \delta \tau+\frac{C v}{\xi_{0}^{3} N^{1 / 2}} \sqrt{N r}
$$


where $\delta \tau \equiv \tau-\tau_{c}$, with $C<0$. The theory exhibits strongly non-classical critical behavior for values of $\delta \tau$ less than a crossover value $\delta \tau^{*}$. Approximating $\delta \tau^{*}$ by the value at which the $\sqrt{r}$ fluctuation correction is equal to $\delta \tau$ yields a reduced crossover temperature

$$
N \delta \tau^{*} \sim \bar{N}^{-1},
$$

as first noted by de Gennes $\frac{17}{}$ As noted by Holyst and Vilgis ${ }^{6}$, the predicted $\mathcal{O}(1 / N)$ with of the critical region is thus much less than the predicted $\mathcal{O}\left(N^{-1 / 2}\right)$ magnitude of the shift of the critical temperature from its SCF value.

Very close to the critical point, $\tau \ll \tau^{*}$, the Hartree theory yields $r \propto \tau^{2}$, or $S(q=0) \propto \tau^{-2}$. The predicted critical exponent of $\gamma=2$ is much larger than the known Ising critical exponent of $\gamma \simeq 1.26$. This is a well known defect of the Hartree theory.

\section{Off-Critical Blends}

At any composition except the critical composition, the Hartree theory considered here does not have a spinodal: At off-critical compositions, because $\chi^{*}$ diverges like $r^{-1 / 2}$ with decreasing $r$, there is no value of $\tau$ for which $r=0$. As noted by Wang $\underline{9}$, in the Hartree approximation, this causes $\chi_{e}$ to become a non-monotonic function of $\chi_{a}$ or (equivalently) $\chi_{a}$ to become a multi-valued function $\chi_{e}$. An example of this behavior is shown in Fig. 3, which shows predictions of renormalized Hartree theory for $\chi_{a}$ vs. $\chi_{e}$ in a series of off-critical blends of various compositions with $b_{1}=b_{2}, N_{1}=N_{2}$, and $\bar{N}=4000$. The turning point at which $\chi_{e}$ reaches its maximum value, which is also where $S(q=0)$ is maximum, is what Wang referred to as a "pseudo-spinodal". We do not attach any physical significance to this turning point, except as a particularly obvious failure of the Hartree approximation.

i

\section{SINGLE CHAIN PROPERTIES}

The one-loop theory 10,18 also predicts slight deviations from random-walk statistics for individual chains. The one-loop approximation for the intramolecular correlations function is a form of renormalized perturbation theory in which each pair of monomers on a chain interact via Edwards' screened interaction, and in which the effect of this interaction is taken into account to first order in powers of the strength of the screened interaction ${ }^{19}$.

We consider the single-chain correlation function $\omega_{i}(\mathbf{k})$. This is given for a Gaussian homopolymer by

$$
\omega_{i}(\mathbf{k})=N^{2} g\left(k^{2} R_{g, i}^{2}\right),
$$

where $R_{g, i}=\sqrt{N_{i} b_{i}^{2} / 6}$ is the radius of gyration of species $i$ and $g(x)=2\left(e^{-x}-1+x\right) / x^{2}$ is the Debye function.

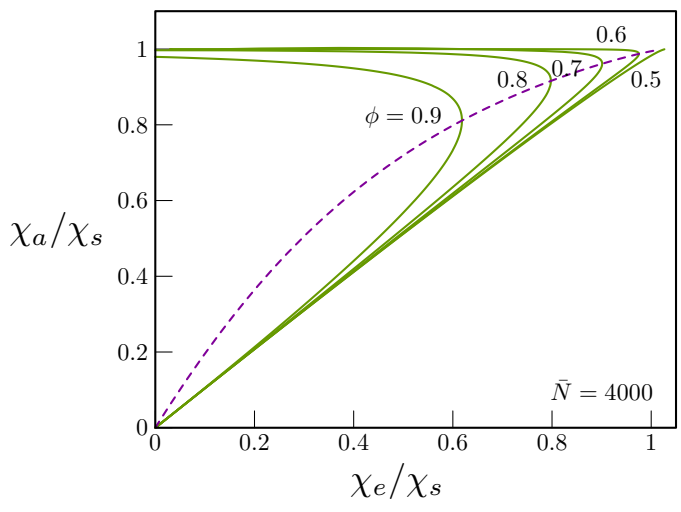

FIG. 3: Self-consistently computed $\chi_{a}$ versus $\chi_{e}$ for off-critical blends with $b_{1}=b_{2}$ and $N_{1}=N_{2}$ and $\bar{N}=4000$ at various values of $\phi=\phi_{1}$. Both axes are rescaled by the spinodal value $\chi_{s}$. Note that the spinodal line $\chi_{a} / \chi_{s}=1$ is actually reached only at the critical composition of $\phi_{1}=1 / 2$. The computed locus of turning points is depicted with dashed line.

The one-loop correction to $\omega_{i}(\mathbf{k})$ in a homopolymer blend is given by 10,18

$$
\delta \omega_{i}(\mathbf{k})=-\frac{1}{2} \int d \mathbf{q} \psi_{i}^{(4)}(\mathbf{k},-\mathbf{k}, \mathbf{q},-\mathbf{q}) G_{i i}(\mathbf{q}) .
$$

Here, $G_{i i}(\mathbf{q})$ is the screened interaction between any two $i$ monomers, which is given in an incompressible blend at wavelengths $q \ll \Lambda$ by

$$
G_{i i}=\frac{1-2 v \chi_{e} \Omega_{1} \Omega_{2} \Omega_{i}^{-1}}{\Omega_{1}+\Omega_{2}-2 v \chi \Omega_{1} \Omega_{2}}
$$

The function $\psi_{i}^{(4)}=\psi_{i}^{(4)}(\mathbf{k},-\mathbf{k}, \mathbf{q},-\mathbf{q})$ is given by

$$
\psi_{i}^{(4)}=\omega_{i}^{(4)}(\mathbf{k},-\mathbf{k}, \mathbf{q},-\mathbf{q})-\omega_{i}(\mathbf{k}) \omega_{i}(\mathbf{q}),
$$

where $\omega_{i}^{(4)}$ is the 4-point intramolecular correlation function for an ideal Gaussian chain ${ }^{10}$. It is straightforward to verify that $\delta \omega_{i}(\mathbf{k})$ vanishes in the limit $\mathbf{k}=0$, as required to retain consistency with the requirement that $\omega_{i}(0)=N_{i}^{2}$.

If the high- $q$ behavior of the above integral is regularized introducing a sharp cutoff at $q=\Lambda$, and using Eq. (48) for all $q<\Lambda$, the resulting integral is found to increase linearly with $\Lambda$ as $\Lambda$ is increased. We ${ }^{10}$ and Beckrich et al $\stackrel{11}{\underline{11}}$ have shown, however, that this UV divergence can be absorbed into renormalization of the statistical segment length. After this UV divergent part is subtracted, the remaining UV convergent correction, which we will denote by $\delta \omega_{i}^{*}(\mathbf{k})$ may be used to characterize the deviation from random-walk statistics. More precisely, $\delta \omega_{i}^{*}(\mathbf{k})$ is the deviation of the intramolecular correlation function $\omega_{i}(\mathbf{k})$ from that of a Gaussian chain with a renormalized statistical segment length $b_{\infty, i}$ characteristic of a dense one-component 
liquid of infinitely long chains. The correction $\delta \omega_{i}^{*}(\mathbf{k})$ is found to be smaller than $\omega_{i}(\mathbf{k})$ by a factor proportional to $\bar{N}^{-1 / 2}$, and exhibits a nontrivial dependence on $\mathbf{k}$.

The fractional deviation of the polymer radius gyration is given by (appendix A

$$
\frac{\delta R_{g, i}^{2}}{R_{g 0, i}^{2}}=-\frac{3}{N_{i}^{2} R_{g 0, i}^{2}} \lim _{\mathbf{k} \rightarrow 0} \frac{\partial \delta \omega_{i}(\mathbf{k})}{\partial\left(k^{2}\right)} .
$$

where $R_{g 0, i}^{2} \equiv N_{i} b_{\infty, i}^{2} / 6$ is the prediction for a Gaussian chain with statistical segment length $b_{\infty, i}$. This fractional deviation is proportional to $\bar{N}^{-1 / 2}$.

\section{A. Monodisperse Melt}

We first consider the case of a monodisperse onecomponent melt, which we obtain by setting $\phi_{2}=0$. The one-loop approximation used here reduces in the case of one-component liquids to that investigated by Semenov et al.11,12,20,21. The only difference in our treatment of this case is our use of a numerical integration and renormalization procedure to obtain accurate results for monodisperse polymers.

Fig 4 shows the predicted correction to the single chain correlation function in a monodisperse melt. From the low $\mathbf{k}$ behavior (see appendix $\mathrm{A}$ ) we find that the predicted fractional change of the radius of gyration is

$$
\frac{\delta R_{g}^{2}}{R_{g 0}^{2}}=-\frac{1.42}{\sqrt{\bar{N}}}
$$

We thus predict a contraction of finite chains, relative to a hypothetical Gaussian chain with a statistical segment length $b_{\infty}$, in agreement with the conclusions of Ref. ${ }^{12}$ [see Eq. (20)].

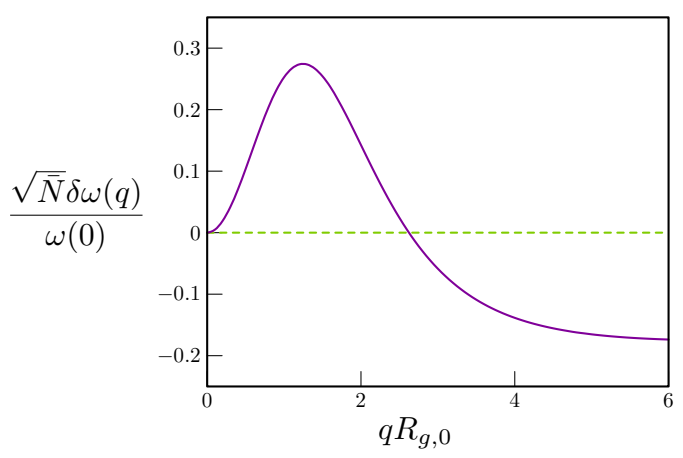

FIG. 4: Predicted correction to the single chain correlation function (Debye function).

\section{B. Bidisperse Melt}

We next consider the behavior of a trace concentration of a species of chains of length $N$ (component 1) in a matrix of chemically homologous shorter chains of length $N_{2}=\alpha N$ (component 2). This limit is obtained by calculating the limit $\phi_{1} \rightarrow 0$ of $\delta \omega_{1}(q)$. To represent chemically similar species, we set $l_{1}=l_{2}$ and $\chi_{e}=0$. This case reduces to that of a mono-disperse melt when $\alpha=1$, and to a dilute solution of polymer in oligomeric solvent when $\alpha \ll 1$.

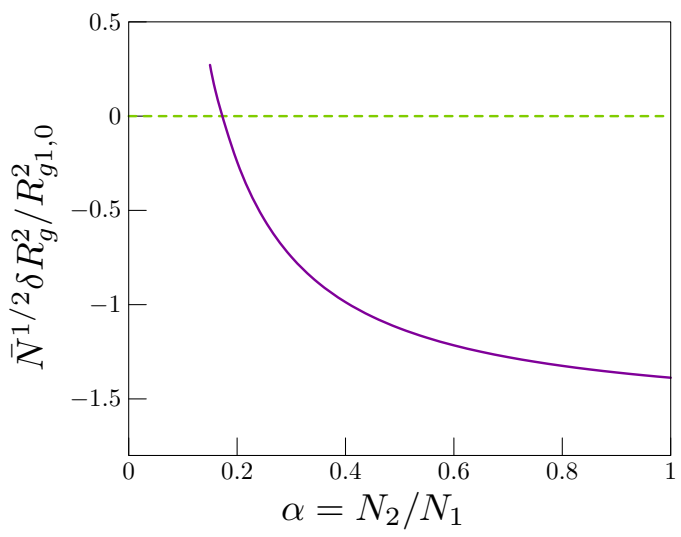

FIG. 5: The fractional change of the radius of gyration of an isolated chain of length $N$ in a matrix of short chains of length $\alpha N$, rescaled with $\sqrt{\bar{N}}$.

In the previous subsection, we noted that the size of a single chain in the pure melt decreases as compared to the unperturbed value. It is also well known that a single chain swells in the dilute solutions with chemically similar small molecule solvents, which create a good solvent. In Fig 5 , we show one-loop predictions for the fractional change of the radius of gyration of a test chain of length $N$ in a matrix of chains of length $\alpha N$. The change is indeed negative for $\alpha$ close to 1 and becomes positive for $\alpha$ close to 0 , passing through zero at $\alpha \simeq 0.17$.

The limit $\alpha \ll 1$ corresponds to a polymer solution in a good small-molecule solvent. The curve in Fig 5 diverges as $0.31 / \alpha$ near $\alpha=0$ (appendix $\mathrm{A}$ ). In this limit, where the matrix chains are point-like, Eq. (48) for the screened interaction between a pair of monomers on a longer chain reduces to a constant $G_{11}(q)=v /(\alpha N)$. Recall that the definition of a "monomer" in an incompressible coarse-grained theory is simply the length of a chain that occupies an arbitrary reference volume $v$. If, to simplify notation, the reference volume is taken to be equal to the excluded volume $v \alpha N$ of a solvent molecule (i.e., a matrix chain), by setting $\alpha N=1$, we obtain an interaction $G_{11}(q)=v$ characterized by an excluded volume parameter equal to the solvent volume. Predictions of the theory in this limit are thus identical to those obtained in the first order perturbation theory for a single chain with a point-like interactions $v \delta(\mathbf{r}){ }^{22}$ It is straightforward to show that this perturbative result is consistent with the prediction $^{23}$ that the longer chain will undergo substantial expansion if $N_{2} \ll \sqrt{N_{1}}$, signalling a breakdown of first order perturbation theory. 


\section{Binary Blends}

We now consider binary blends with $\chi \neq 0$. For simplicity, we focus on symmetric systems, with $N_{1}=N_{2}=N$ and $b_{1}=b_{2}=b$. Fig. 6 shows the one-loop predictions for the fractional change of the radius of gyration squared, rescaled by $\sqrt{\bar{N}}$, as function of $\chi_{e} N$ for various compositions. Increasing $\chi$ always causes both species in a blend to contract. The dependence of $R_{g 1}$ upon $\chi$ is strongest when species 1 is a tracer in a matrix of $2\left(\phi_{1} \ll 1\right)$. There is no dependence of $R_{g 1}$ upon $\chi$ in the opposite limit $\phi_{2} \ll 1$ of essentially pure homopolymer 1 . In the dilute limit, $\phi_{1} \rightarrow 0$, the one-loop theory predicts a deviation $\delta R_{g 1}^{2}$ that is a strictly linear function of $\chi N$. Furthermore, it can be shown that the slope of the limiting line is -0.62 , which is -2 times that of the coefficient to the $1 / \alpha$ divergence for the bidisperse melt in the limit of small-molecule good solvent discussed in the previous subsection (appendix A).

It is interesting to note that at the critical composition, $\phi_{1}=1 / 2$, the deviation induced by increasing $\chi$ always remains less than the chain-length dependent deviation from ideal chain statistics that is already present in the onecomponent melt $(\chi=0)$. Also note that the radius of gyration is a smooth, nearly linear function of $\chi$ even near the critical point. This reflects the fact that the long-wavelength composition fluctuations that appear near the critical point have characteristic wavelengths much larger than the radius of gyration, and so couple very weakly to the conformations of individual chains.

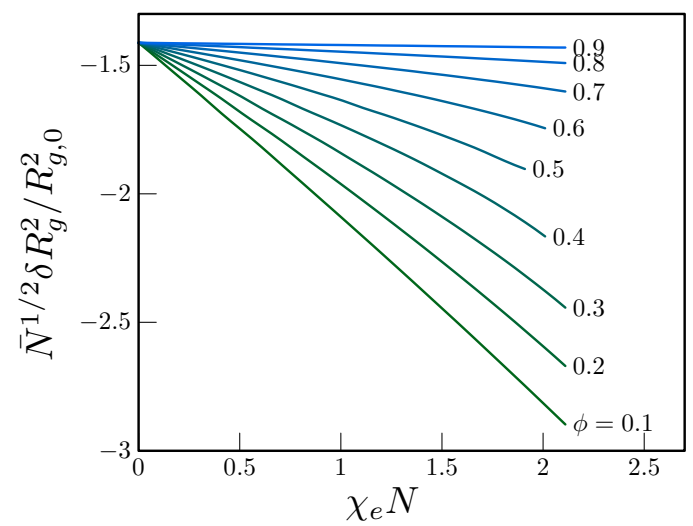

FIG. 6: The changes in the radius of gyration of chains at various compositions plotted as functions of $\chi_{e} N$ in symmetric binary blends. The chains become more contracted as $\chi_{e} N$ increases and the effects is more pronounced for minority component. In the limit of $\phi=1$, the change becomes independent of $\chi_{e} N$; In the limit of $\phi=0$, the change is linear in $\chi_{e} N$, as can be told from the functional form of $G_{i}$, Eq.47.

\section{COMPARISON TO SIMULATION}

Here, we compare the predictions of the one-loop theory to the results of lattice Monte-Carlo simulation. We compare to bond-fluctuation model (BFM) simulations of symmetric blends at $\phi_{1}=1 / 2$ Binder, Deutsch, and Müller, and to BFM simulations of single-chain properties by Beckrich et al.11. The bond fluctuation model is a lattice model on a simple cubic lattice in which each monomer occupies a set of 8 sites at the vertices of a cube. In all of the simulations discussed here, the monomer concentration is 1/16 monomer per lattice site, which is half the maximum possible concentration in this model. The statistical segment length extrapolated for the infinitely long chain is 3.244 , which has been reported in ${ }^{12}$. In simulations of polymer blends, monomers of types $i$ and $j$ on neighboring sites interact with an energy $u_{i j}$, with $u_{11}=u_{22}$ and $u_{12}>u_{11}$. Binder and coworkers have carried out semigrand canonical ensemble simulations using two different variants of the rules for what constitute a "neighbor": One in which each monomer can interact only with monomers located at the 6 closest allowed sites, and another in which each monomer can interact monomers with monomers at any of the 54 closest allowed sites.

\section{A. Determining $\chi_{e}$}

To compare predictions of a coarse-grained theory to simulation data, one must somehow establish a relationship between the interaction parameters used in the simulations and the phenomenological interaction parameter used in both Flory-Huggins theory the one-loop theory. In an accompanying paper, we propose a way to do this, which we use here. The analysis given there refines and justifies a form of "modified Flory-Huggins theory" that was originally proposed by Müller and Binder ${ }^{24}$ There, we consider a perturbation theory for a lattice model of the type described above, in which free energy of mixing is expanded in powers of a perturbation parameter $\alpha=u_{12}-u_{11}$. We identify the SCF interaction free energy per monomer $f_{\text {int }}$ by considering the behavior of the free energy of mixing in the limit of infinitely long chains. We show there that $f_{\text {int }}$ is given to first order in a power series in $\alpha$ by a function

$$
f_{\text {int }}\left(\epsilon, \phi_{1}\right)=k T \chi_{e} \phi_{1}\left(1-\phi_{1}\right)
$$

in which

$$
\chi_{e}=z^{\infty}\left[u_{12}-u_{11}\right] / k T
$$

Here, $z^{\infty}$ is an "effective coordination number" that is obtained by evaluating a value $z(N)$ of the average number of inter-molecular nearest neighbors per monomer in a one-component reference liquid, with $\alpha=0$, and extrapolating the value to the limit of infinitely long chains. The values of $z^{\infty}$ used here were reported by Müller and 
Binder: $z_{c}^{\infty}=2.1$ for the model with 54 interacting sites, and $z_{c}^{\infty}=0.307$ for the model with 6 interacting sites. A more complete discussion of the reasoning underlying this prescription is given elsewhere.

Our use of a first order perturbation theory to estimate $f_{\text {int }}$ in the one-phase region of the phase diagram is justified by the fact that the critical value of $\alpha$ decreases as $1 / N$ with increasing chain length. The fractional error in our estimate of $\chi_{e}$ arising from the use of a first order expansion is thus expected to be of order $1 / N$ near the critical point. For $N \gg 1$, this error is thus expected to be much smaller than the corrections to $\chi_{a}$ predicted by the one loop theory, which are of order $N^{-1 / 2}$. The resulting $\mathcal{O}(1 / N)$ errors in our estimate of the "bulk" interaction parameter $\chi_{e}$ are expected to be comparable in importance to the $\mathcal{O}(1 / N)$ corrections that arise from end effects.

\section{B. Composition Fluctuations in Blends}

Fig. 7 shows the results of Müller for $N S^{-1}(0) / 2$ plotted vs. $\chi_{e} N$ for bond-fluctuation model simulations of chains of length $N=64^{25}$. These simulation used a variant of the BFM in which interactions extend over 54 neighboring sites, and in which $u_{11}=-u_{12}$. Values of $S^{-1}(0)$ were extracted from the semi-grand equation of state. We have calculated the SCF effective $\chi_{e}$ parameter for each simulation from Eq. (52) using the value of $z^{\infty}=2.1$ reported by Mueller and Binder. 24

In the upper panel of Fig. 7 the solid line is the RPA prediction and the dashed line is the one-loop prediction obtained using the appropriate value of $\bar{N}=290$ (The value was reported as 240 , which were computed from the statistical segment length of $N=64$ chains). The lower shows a comparison of simulation results for the difference $\chi^{*} N=\left(\chi_{a}-\chi_{e}\right) N$ multiplied by $\sqrt{\bar{N}}$ to the universal curve shown in Fig. 2 We see that the self-consistent oneloop theory gives an extremely accurate description of deviations from SCFT far from the critical point, for $\chi_{a} N \lesssim 1$, but appears to overestimate the stabilizing effect of critical fluctuations. For this system, the suppression of $\chi_{a}$ by long-wavelength fluctuations near the critical point is almost exactly cancelled by the enhancement of $\chi_{a}$ at smaller values of $\chi_{e}$, giving a critical value of $\chi_{e} N$ that is closer to the SCFT value than to the value predicted by the one-loop theory.

Fig. 8 compares the predictions of the one-loop theory for the ration $T_{c}^{\infty} / T_{c}(N)$ for different values of $N$ to the results of simulations by Deutsch and Binder ${ }^{26}$. Here, $T_{c}^{\infty} \equiv \alpha z^{\infty} N /(2 k)$ is the critical temperature that is predicted by SCF if we use Eq. (52) for $\chi_{e}$. The critical temperatures reported by Deutsch and Binder were identified for each chain length by a finite-size scaling analysis of simulations with different simulation cell sizes. Simulation results are shown for variants of the BFM model in which the neighbor interactions extend over 6 neighbors (circles)
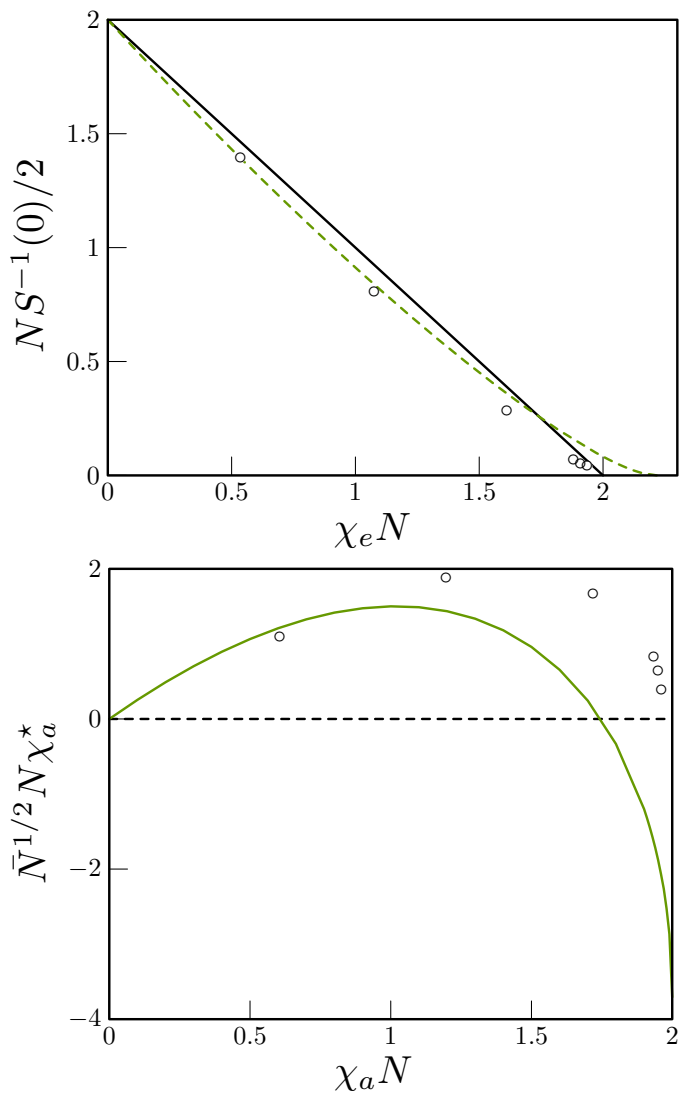

FIG. 7: Comparison of the results of Müller's bond-fluctuation model lattice simulations $N S^{-1}(0) / 2$ (circles) to corresponding one-loop 1-loop predictions (dashed line), for $N=64$ and $\bar{N}=$ 290 , for a half-filled lattice ${ }^{25}$. The calculation of values of $\chi_{e}$ for simulation results is discussed in the text.

and over 54 neighbors (triangles), For both versions of the models, $T_{c}^{\infty} / T_{c}(N)$ extrapolates to unity as $N \rightarrow \infty$. This confirms that first order perturbation theory prediction for $T_{c}$ is indeed asymptotically exact in the limit $N \rightarrow \infty$.

For both versions of the BFM, however, the measured deviations of $T_{c}^{\infty} / T_{c}(N)$ are significantly smaller than those predicted by the one-loop theory. This quantitative discrepancy must be, in part, a result of the inadequacy of the one-loop theory as a theory of critical phenomenon, and the need for a renormalization group approach near the critical point. This data suggests that, if it is indeed possible to express universal corrections to $T_{c}^{\infty} / T_{c}(N)$ that arise from critical fluctuations as a power series in $1 / \sqrt{N}$, the coefficient of any $\mathcal{O}\left(N^{-1 / 2}\right)$ correction must be much smaller than predicted by the one-loop theory.

It is important to also note that the slight deviations of $T_{c}^{\infty} / T_{c}(N)$ from unity in Fig. 8 are not the same for the two variants of the BFM, and appear to of opposite sign. This model-dependence cannot be so easily explained as a failure of the one-loop approximation near the critical point, since we expect that a more sophisticated treatment 
of critical phenomena in a simple coarse-grained model would also predict a universal dependence of $T_{c}^{\infty} / T_{c}(N)$ on the dimensionless parameter $\bar{N}$. The observed nonuniversality could, however, be the result of corrections arising from contribution to $f_{\text {int }}$ that are second order in $\alpha$, which are neglected in our estimate of $\chi_{e}$, and/or the result of end effects. Both of these effects are neglected in our analysis, and both are expected to yield model dependent corrections to $T_{c} / T_{c}^{\infty}$ that are proportional to $1 / N$. It appears to us that the dominant contribution to the deviation $T_{c}^{\infty} / T_{c}(N)-1$ for this data may well be a nonuniversal correction proportional to $1 / N$ (which appears to provide a better description of the $N$ dependence, as originally suggested by Deutsch and Binder) rather than a universal correction proportional to $1 / \sqrt{\bar{N}}$.

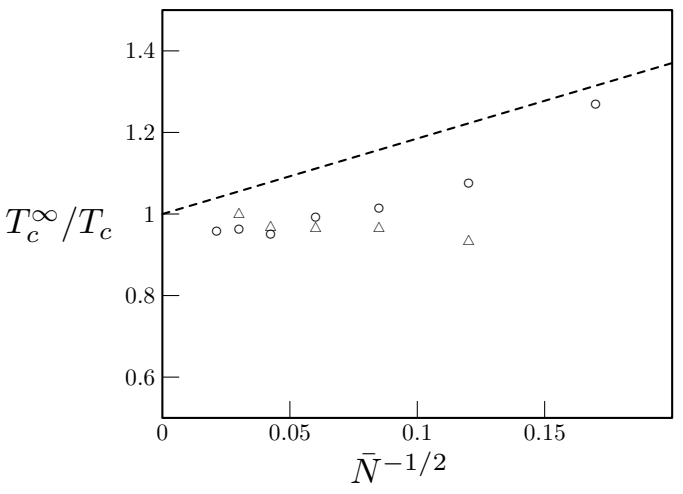

FIG. 8: Chain length dependence of the shift in the critical temperature. Comparison between 1-loop theory prediction and Deutsch and Binder's lattice simulation ${ }^{26}$. Simulation results are shown for variants of the BFM in which interactions extend over 6 nearest neighbors (circles) and 54 neighbors (triangles).

\section{Single-Chain Correlations}

In Fig 9 we compare one-loop predictions and simula-

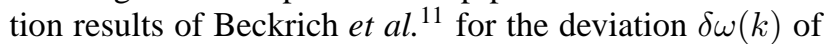
the correlation function for a monodisperse melt from the Debye function. Simulation results for 5 different chain lengths were taken from Ref. ${ }^{11}$. As noted by Beckrich et $a l$. , the collapse of data for from different chain lengths when $\sqrt{\bar{N}} \delta \omega(q)$ is plotted vs. $k R_{g 0}$ already shows that the deviation is proportional to $1 / \sqrt{\bar{N}}$, as predicted by the one-loop theory. Simulations and predictions agree quite well, though there do appear to be some small systematic discrepancies in the high $\mathbf{k}$ regime. The agreement is somewhat better than that obtained by the Strasbourg group using a more approximate treatment of a monodisperse melt. A similar level of agreement was obtained previously by that group in comparisons of the one-loop theory to simulations of polydisperse equilibrium polymers, for which they obtained analytic predictions for $\delta \omega(q) .11,12,20,21,27$.

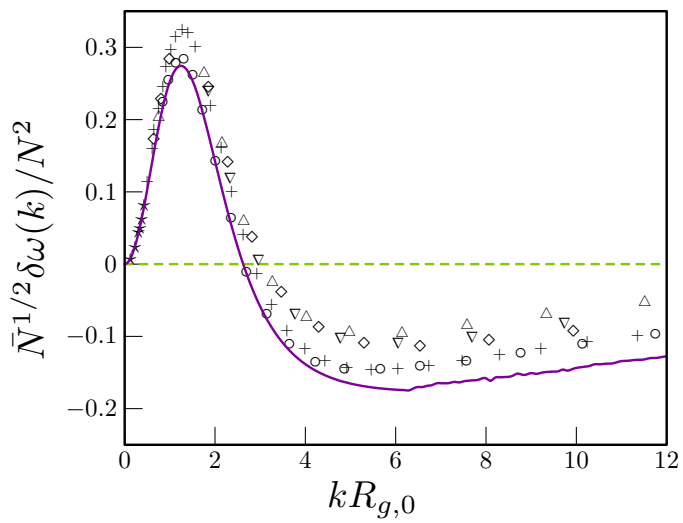

FIG. 9: One-loop correction to the single chain correlation function (Debye function). The data points are gleaned from Fig.6 of Ref. ${ }^{11}$. Different symbols are lattice simulation $(\rho=1 / 16$, $b \simeq 3.24)$ results for chains with different lengths: $+(N=2048)$, $\circ(1024), \diamond(512), \nabla(256), \triangle(128)$. Near $k=0$ the data points for various chain lengths overlap and can not be distinguished from the original figure, so are symbolized as $\star$.

Müller has examined the dimension of homopolymer chains of various lengths in binary blends with varying compositions and interactions ${ }^{28}$, using Monte Carlo simulations of the BFM with the same parameters as the one used to study composition fluctuations ${ }^{25}$. In Fig 10 we replotted $\sqrt{\bar{N}}$ times his results for the fractional deviation of the mean-squared end-to-end vector $R^{2}$ for component $A$ over a range of values of $\phi=\phi_{A}$ for a fixed value $\alpha N / k_{B} T=0.32$, corresponding to $\chi_{e} N=0.672$, for 6 different chain lengths, adapted from Fig. $5 \mathrm{~b} \mathrm{in}^{28}$. Here $\delta R^{2}$ represents the deviation of $R^{2}$ in a mixture from the ideal Gaussian chain value calculated using the statistical segment length of a sufficiently long chain. The original results were presented as the difference between the value in the blend and that in a melt of chains of the same length. We have switched the normalization by assuming that the radius of gyration measured in the melt is smaller compared to the ideal value by the amount $1.42 / \sqrt{\bar{N}}$. The fact that results for different chain lengths collapse confirms by itself that these deviations are proportional to $1 / \sqrt{N}$. The method of obtaining the theoretical curve in Fig 10 is sketched in appendix $B$. The qualitative trend of the simulation results were relatively well described with the theory.

\section{THERMODYNAMIC INTEGRATION OF THE RPA}

The one-loop approximation has most often been presented within the context of the Edwards formalism, as a Gaussian approximation for the functional integral representation for the partition function. Here, we discuss an alternative derivation that makes no reference to the Edwards formalism, in which the one-loop free energy is obtained by thermodynamic integration with respect to the strength 


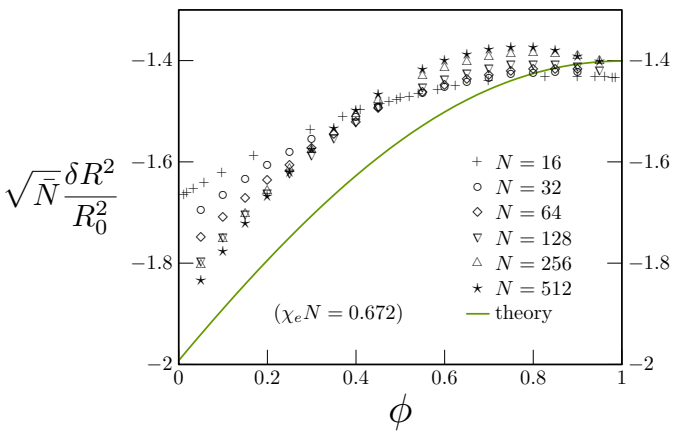

FIG. 10: Composition dependence of the fractional change of the mean squared end-to-end distance for homopolymer chains in a binary blends for systems with $\chi_{e} N=0.672$ and variable composition. Simulation data were adapted from Müller's data for $\left\langle R^{2}\right\rangle^{28}$. Solid line is the one-loop prediction.

of either the pair potential or the bare interaction parameter, while using the RPA approximation for $S_{i j}(q)$. The derivation is closely analogous to the usual derivation of the Debye-Hückel approximation for excess free energy of an electrolyte solutions, which is obtained by combining an expression for the free energy as an integral with respect to a "charging" parameter with the Debye-Hückel linear selfconsistent field approximation for the correlation function (see, e.g., McQuarrie ${ }^{29}$, pgs. 328-339).

\section{A. Variation of the Pair Potential}

Our analysis starts with the following exact expression for the dependence of the free energy $F=-\ln Z$ upon the pair potential $U_{i j}\left(\mathbf{r}-\mathbf{r}^{\prime}\right)$ in any model with a pair potential: The perturbation $\delta F$ in the free energy induced by an infinitesimal perturbation $\delta U_{i j}\left(\mathbf{r}-\mathbf{r}^{\prime}\right)$ in the pair potential is given by an integral

$$
\delta F=\frac{1}{2} \int d \mathbf{r} \int d \mathbf{r}^{\prime}\left\langle c_{i}(\mathbf{r}) c_{j}\left(\mathbf{r}^{\prime}\right)\right\rangle \delta U_{i j}\left(\mathbf{r}-\mathbf{r}^{\prime}\right)
$$

The corresponding perturbation of the correlation free energy density $f_{\text {corr }}$ may expressed as an integral

$$
\delta f_{\text {corr }}=\frac{1}{2} \int d \mathbf{r} S_{i j}(\mathbf{r}) \delta U_{i j}(\mathbf{r})
$$

or as a Fourier integral

$$
\delta f_{\text {corr }}=\frac{1}{2} \int_{\mathbf{q}} S_{i j}(\mathbf{q}) \delta U_{i j}(\mathbf{q}),
$$

where $S_{i j}(\mathbf{r}) \equiv\left\langle c_{i}(\mathbf{r}) c_{j}(0)\right\rangle-\left\langle c_{i}\right\rangle\left\langle c_{j}\right\rangle$,

By explicitly evaluating the corresponding variation of Eq. (10) for the one-loop correlation free energy for a compressible liquid, we find that, in this approximation,

$$
\delta f_{\text {corr }}=\frac{1}{2} \int_{\mathbf{q}} S_{i j}^{\mathrm{RPA}}(\mathbf{q}) \delta U_{i j}(\mathbf{q})
$$

where

$$
S_{i j}^{\mathrm{RPA}}(\mathbf{q})=\left[\Omega^{-1}(\mathbf{q})+U(\mathbf{q})\right]_{i j}^{-1}
$$

is the RPA (or mean-field) approximation for $S_{i j}(\mathbf{q})$ in a compressible liquid. We thereby find that the one-loop approximation for the free energy density is thermodynamically consistent with an RPA approximation for $S_{i j}(\mathbf{q})$.

It follows that the one-loop free energy may be obtained by a thermodynamic integration similar to that used to obtain the free energy in the Debye-Hückel theory of electrolytes: Consider a sequence of systems with a reduced pair potential $\lambda U_{i j}$, with a charging parameter $\lambda$ in the range $0<\lambda<1$. The one-loop correlation energy (which vanishes in the $\lambda=0$ ideal gas state) may be expressed as an integral

$$
f_{\text {corr }}=\frac{1}{2} \int_{0}^{1} d \lambda \int_{\mathbf{q}} U_{i j}(\mathbf{q}) S_{i j}^{R P A}(\mathbf{q} ; \lambda)
$$

in which $S_{i j}^{R P A}(\mathbf{q} ; \lambda)$ is the RPA correlation function for a compressible mixture with a reduced pair potential $\lambda U_{i j}$. It is straightforward to confirm that Eq. (10) is recovered by explicitly completing the integral with respect to $\lambda$.

\section{B. Variation of $\chi_{0}$}

For many purposes, it is useful to consider the simple special case of a symmetric mixture of structurally identical chains, with $b_{1}=b_{2}$ and $N_{1}=N_{2}$, with a pair potential of the form given in Eq. (2). In this case, the limit $\chi_{0}=0$ yields a true ideal mixture, for which $f_{\text {corr }}$ is independent of composition. In a nearly-incompressible symmetric mixture, with $B v \gg 1$, the composition dependence of $f_{\text {corr }}$ may thus be obtained by starting from a nearly incompressible reference state with $\chi_{0}=0$, and calculating how $f_{c o r r}$ varies as $\chi_{0}$ is increased. As a special case of Eq. (55), it is straightforward to show that

$$
\frac{\partial f_{\text {corr }}}{\partial \chi_{0}}=v \int d \mathbf{r} S_{12}(\mathbf{r}) \delta_{\Lambda}(\mathbf{r})
$$

If we take the incompressible limit, for which $S_{12}(\mathbf{r})=$ $-S(\mathbf{r})$, and the limit $\Lambda \rightarrow \infty$ of point-like interactions, Eq. (61) reduces to

$$
\lim _{\Lambda \rightarrow \infty} \frac{\partial f_{\text {corr }}}{\partial \chi_{0}}=-v \lim _{\mathbf{r} \rightarrow 0} S(\mathbf{r}),
$$

or

$$
\frac{\partial f_{\text {corr }}}{\partial \chi_{0}}=-v \int_{\mathbf{q}} S(q)
$$

in Fourier space. 
The one-loop theory may be obtained by using the incompressible RPA approximation for $S(q)$ in Eq. (61). In a symmetric blend, the incompressible RPA yields

$$
S^{R P A}(q ; \chi)=\frac{\phi_{1} \phi_{2}}{\Omega^{-1}(q)-2 v \chi \phi_{1} \phi_{2}} .
$$

An idealized IRPA model of Gaussian chains with pointlike interactions, however, yields a UV divergent integral in Eq. (61). This may be regularized by restricting the integral to $q<\Lambda$. The regularized (but unrenormalized) one-loop free is then given, to within a composition independent constant, by an integral

$$
f_{\text {corr }}=-v \chi_{0} \int_{0}^{1} d \lambda \int_{\mathbf{q}} S^{R P A}\left(q ; \lambda \chi_{0}\right)
$$

where the Fourier integral is constrained to $|\mathbf{q}|<\Lambda$.

\section{CORRELATION HOLE EFFECTS}

Several features of the one-loop approximation for $f_{\text {corr }}$ may be understood by relating them to corresponding features of the incompressible RPA description of the socalled correlation hole. To show this, it is useful to consider the behavior of the derivative $\partial f_{\text {corr }} / \partial \chi_{0}$ in the simple case of an ideal statistical mixture, which is obtained in our model by setting $b_{1}=b_{2}, N_{1}=N_{2}$, and $\chi_{0}=0$. In this limit, Eq. (62) for $S(q)$ reduces to

$$
S(q)=\phi_{1} \phi_{2} \Omega(q)
$$

This expression can also be obtained more directly by the following physical arguments: Let $S(r)=\langle\delta c(r) \delta c(0)\rangle$ be the overall correlation function in a one-component reference liquid of either pure 1 or pure 2 , and let $H(r)$ and $\Omega(r)$ be the inter-molecular and intramolecular correlation functions, so that $S(r)=\Omega(r)+H(r)$. An ideal mixture may be created by randomly labelling a fraction $\phi_{1}$ of the chains in such a one-component liquid as species " 1 ", and the remainder as species " 2 ". In the resulting ideal mixture, the probability that a particular pair of monomers on different chains will be on chains labelled $i$ and $j$ is simply $\phi_{i} \phi_{j}$. It follows that the inter-molecular correlation function $H_{i j}(r)$ in such a mixture is simply $H_{i j}(r)=\phi_{i} \phi_{j} H(r)$. Similarly, because the probability that two monomers on the same chain belong to a chain of type $i$ is $\phi_{i}$, the intra-chain correlation function $\Omega_{i j}(r)$ in such a mixture is $\Omega_{i j}(r)=$ $\delta_{i j} \phi_{i} \Omega(r)$. Thus far, everything we have said is exact.

In the idealized limit of a nominally incompressible liquid, however, we assume that $S(r)=0$, and thus that $\Omega(r)+H(r)=0$. In an incompressible liquid, the interchain correlation function $H(r)$ must thus have a "correlation hole", which is a mirror image of the intra-chain correlation function $\Omega(r)$. This implies that, in an incompressible statistical mixture, $H_{i j}(q)=-\phi_{i} \phi_{j} \Omega(q)$.
Using this assumption, it is straightforward to show that $S_{12}(q)=-\phi_{1} \phi_{2} \Omega(q)$ and that $S_{11}(q)=+\phi_{1} \phi_{2} \Omega(q)$ in any incompressible ideal mixture.

\section{A. UV divergence (Unrenormalized Theory)}

The UV divergence of the correlation correction to $\chi_{e}$ in Eq. (18) is the result of a pathological feature that arises from a combination of the assumption of rigorous incompressibility, at all wavelengths, in a model of continuous Gaussian chains. In the limit $\chi_{0}=0$ and $\Lambda \rightarrow \infty$, Eqs. (61) and 64 may be combined to show that

$$
\frac{\partial f_{\text {corr }}}{\partial \chi_{0}}=v \phi_{1} \phi_{2} H(r=0)=-v \phi_{1} \phi_{2} \Omega(r=0),
$$

In the limit $\Lambda \rightarrow 0$, the predicted free energy is sensitive to the value of $H(r)$ only at $r=0$ because the range of the pair interaction $U_{i j}$ vanishes in this limit. It is well known, however, that the intramolecular correlation function $\Omega(r)$ for a continuous Gaussian thread diverges as $\Omega(r) \propto 1 / r$ as $r \rightarrow 0$. Recall the origin of this divergence: The number of monomers within a region of volume $r^{3}$ near a test monomer is of order the number of monomers $g$ in a segment of chain of size $r=\sqrt{g} b$, giving an average concentration $g / r^{3} \sim 1 /\left(r b^{2}\right)$ within a region of size $r$, or $\Omega(r) \propto 1 / r$. When combined with an assumption of rigorous incompressibility, however, the divergence in $\Omega(r)$ yields an infinitely deep correlation hole in $H(r)$ at $r=0$ in the one-component reference liquid. Taken literally, the incompressible RPA would yield negative values of $\langle c(r) c(0)\rangle$ in a region around $r=0$. This leads to the nonsensical prediction of a divergent reduction in $H(r=0)$, and thus to a divergent negative correction to $\chi_{e}$.

The UV divergence obtained from the IRPA that underlies the one-loop theory could be avoided by the use of any model that avoids the use of any of: i) a point-like interaction, ii) a continuous Gaussian thread model, or iii) rigorous incompressibility. For example, in the thread limit of the PRISM theory ${ }^{30}$, one retains a model of continuous Gaussian chains, and a point-like effective interaction (i.e., direct correlation function), but avoids complete nonsense by allowing for some compressibility at short wavelengths.

\section{B. $\quad N$-dependence (Renormalized Theory)}

In the renormalized one-loop theory, the UV divergent part of the correlation correction to $\chi_{a}$ is absorbed into the definition of $\chi_{e}(\Lambda)$. Once the divergence is removed, the renormalized theory can make concrete predictions about the difference $\chi^{*}=\chi_{a}-\chi_{e}$. Because the one-loop expression for $\chi^{*}$ is proportional to $\bar{N}^{-1 / 2}, \chi_{e}$ may be interpreted as the limit of $\chi_{a}$ extrapolated to the limit $N \rightarrow \infty$. We now show that the value of $\chi^{*}$ is sensitive to the slight 
difference between the depth of the correlation hole in a system of finite chains and that in a hypothetical system of infinite chains.

To show this, consider the derivative of Eq. (20) for $f^{*}\left(\chi_{a}\right)$ with respect to $\chi_{a}$, evaluated (for simplicity) in a reference state with $\chi_{a}=\chi_{0}=0$. A straightforward differentiation yields

$$
\lim _{\chi_{a} \rightarrow 0} \frac{\partial f^{*}}{\partial \chi_{a}}=-v \phi_{1} \phi_{2} \int_{\mathbf{q}}^{*} \Omega(q) .
$$

The only differences between Eq. (66) and the limit $\chi_{0}=0$ of Eq. (61) for $\partial f_{\text {corr }} / \partial \chi_{0}$ are: i) the use of a renormalized integral (denoted by an asterisk) to remove the UV divergence, ii) the use of $\chi_{a}$ rather than $\chi_{0}$ as the input parameter in a Hartree approximation.

The UV divergent part of the integral in Eq. (66) is given by a difference

$$
\lim _{\chi_{a} \rightarrow 0} \frac{\partial f}{\partial \chi_{a}}=-v^{2} \phi_{1} \phi_{2} \int_{\mathbf{q}}\left[\Omega(q)-\Omega^{\Lambda}(q)\right],
$$

in which $\Omega^{\Lambda}(q)$ is an asymptotic approximation for $\Omega(q)$ in the limit $q \gg R^{-1}$. This quantity is given by

$$
\Omega^{\Lambda}(q) \equiv 12 /\left(v q^{2} b^{2}\right)
$$

Let $\Omega(q ; N)$ denote the intramolecular correlation function for a single-component melt of chains of length $N$. Because $\Omega^{\Lambda}(q)$ is an asymptotic expansion of $\Omega(q ; N)$ in the limit $q R \gg 1$, it may be interpreted equally well as either:

i) The high- $q$ behavior of $\Omega(q ; N)$ for chains of fixed length $N$, or

ii) The asymptotic behavior of $\Omega(q ; N)$ at fixed $q$ in the limit $N \rightarrow \infty$.

By adopting the latter interpretation, and inverting the Fourier transform, Eq. 67, may thus be expressed as a difference

$$
\lim _{\chi_{a} \rightarrow 0} \frac{\partial f^{*}}{\partial \chi_{a}}=-v \phi_{1} \phi_{2} \lim _{\mathbf{r} \rightarrow 0}[\Omega(\mathbf{r} ; N)-\Omega(\mathbf{r} ; \infty)]
$$

or, equivalently,

$$
\lim _{\chi_{a} \rightarrow 0} \frac{\partial \chi^{*}}{\partial \chi_{a}}=v^{2} \lim _{\mathbf{r} \rightarrow 0}[H(\mathbf{r} ; N)-H(\mathbf{r} ; \infty)]
$$

where $H(\mathbf{r} ; N)$ denotes the value of the inter-molecular correlation function $H(\mathbf{r})$ in a one-component melt of chains of length $N$.

The renormalization procedure that was originally introduced remove the UV divergence is thus seen to be equivalent to the subtraction of the $N \rightarrow \infty$ limit. The resulting difference relates $\chi^{*}=\chi_{a}-\chi_{e}$ to the difference in the depth of the correlation hole at $\mathbf{r}=0$ in a system of finite chains and that in a hypothetical system of infinite chains. The renormalized coarse-grained theory correctly predicts the behavior for $\chi^{*}$ for small values of $\chi_{a}$ because it correctly describes this subtle difference between finite and infinite chains, despite the fact that it predicts a UV divergence $H(\mathbf{r} ; N)$ itself.

\section{CONCLUSIONS}

We have use the renormalized one-loop theory to analyze corrections to the RPA description of composition fluctuations in binary blends, and to the random walk model of chain conformations.

Our treatment of long-wavelength composition fluctuations is closely related to an earlier treatment by Wang, who focused primarily on behavior near the spinodal 9 Our predictions can be summarized by considering the difference $\chi^{*} \equiv \chi_{a}-\chi_{e}$ between an apparent interaction parameter $\chi_{a}$ that would be inferred from the magnitude $S(q=0)$ of long-wavelength composition fluctuations and a parameter $\chi_{e}$ that we identify as the SCFT interaction parameter, which is the limit of $\chi_{a}$ as $N \rightarrow \infty$.

We show that the deviation from SCFT can be understood as the result of two competing physical effects: In the limit $\chi_{e} N \ll 1$ of a nearly ideal mixture, $\chi_{a}$ is greater than $\chi_{e}$ because of a simple packing effect: Monomers on a finite chain are slightly less strongly shielded from contact with monomers of other chains than are monomers on an infinite chain, and are therefore more exposed to unfavorable interactions with chains of the other species in a binary blend. We discuss this effect from another point of view in an accompanying paper. Closer to the spinodal, however, the build up of long-wavelength correlations causes the difference $\chi_{a}-\chi_{e}$ to decrease with increasing $\chi_{e}$. Near the critical point, both effects yields contributions to $\chi_{a}$ that are proportional to $\bar{N}^{-1 / 2}$, and comparable in magnitude. An accurate prediction of the magnitude and sign of the absolute shift in the critical value of $\chi_{e} N$ thus requires an accurate description of both of these competing effects. A simple renormalized Hartree theory predicts an overall enhancement in the critical value $\left(N \chi_{e}\right)_{c}$ (or a depression in $T_{c}$ ) for a symmetric blend by an amount proportional to $\bar{N}^{-1 / 2}$, as first suggested by Holyst and Vilgis ${ }^{6,7}$

Comparison to the data from lattice Monte Carlo simulations of symmetric blends at the critical composition indicate that the one-loop theory gives an excellent description of the packing effect that dominates corrections to the RPA for $\chi_{e} N \lesssim 1$, but a mediocre description of correlation effects closer to the critical point. It appears that the selfconsistent one-loop (or Hartree) theory gives a reasonable semi-quantitative description of deviations from SCFT, but tends to overestimate the magnitude of the depression in $\chi_{a}$ near the critical point, and thus to predict too high a value for $\left(\chi_{e} N\right)_{c}$.

Predictions for corrections to random-walk statistics were also compared to the available simulation data. Predictions for deviations of the intramolecular correlation function from the Debye function for chains in a monodisperse single-component melt agree very well with the simulation results of Beckrich et al., 11 and deviate only slightly from a more approximate theoretical treatment of monodisperse polymers by the same group. Predictions of the composition dependence of the radius of gyration in a binary 
blend with $\chi_{e} \neq 0$ show a similar level of quantitative agreement with the results of lattice Monte Carlo simulations.

\section{APPENDIX A: RADIUS OF GYRATION}

The $k$-dependence of the correction to the intramolecular correlation function of a homopolymer, Eq.47), comes from that of $\psi_{i}^{(4)}(\mathbf{k},-\mathbf{k}, \mathbf{q},-\mathbf{q})$. A straightforward Taylor expansion of $\psi_{i}^{(4)}$ shows that in the low $\mathbf{k}$ regime, to second order in an expansion in powers of $|\mathbf{k}|$,

$$
\psi_{i}^{(4)} \simeq N_{i}^{4} f\left(q^{2} R_{g 0, i}^{2}\right) k^{2} R_{g 0, i}^{2} \cos ^{2} \theta
$$

where $\theta$ is the angle between vectors $\mathbf{k}$ and $\mathbf{q}$, and

$$
\begin{aligned}
f(x) & \equiv \frac{2}{3}\left(\frac{1}{x^{2}}-\frac{24}{x^{4}}-\frac{96}{x^{5}}-\frac{120}{x^{6}}\right) \mathrm{e}^{-x} \\
& +\frac{8}{3}\left(\frac{1}{x^{3}}-\frac{3}{x^{4}}-\frac{6}{x^{5}}+\frac{30}{x^{6}}\right)
\end{aligned}
$$

This expansion has been obtained previously in the studies of chain sizes in semi-dilute solutions ${ }^{14}$ and in melts. ${ }^{31}$ Using the fact that $\omega(\mathbf{k}) \simeq N_{i}^{2}\left(1-k^{2} R_{g, i}^{2} / 3\right)$ in the low-k region and Eq.47, one finds:

$$
\frac{\delta R_{g, i}^{2}}{R_{g 0, i}^{2}}=\frac{3 N_{i}^{2}}{2} \int_{\mathbf{q}}^{*} f\left(q^{2} R_{g 0, i}^{2}\right) \cos ^{2} \theta G_{i i}(\mathbf{q})
$$

This is the general expression used to calculate the fractional change of the radius of gyration.

Consider the variation of $R_{g}^{2}$ for a tracer amount of polymer species 1 in a matrix of chains of species 2 . In the relevant limit $\phi_{1} \rightarrow 0$, the screened interaction $G_{11}$ reduces to:

$$
G_{11}(q)=\frac{v}{N_{2}}\left(\frac{1}{g\left(q^{2} R_{g 0,2}\right)}-2 \chi_{e} N_{2}\right)
$$

where $g(x)=2\left(e^{-x}-1+x\right) / x^{2}$ is the Debye function and $R_{g 0,2}=N_{2} b_{2}^{2} / 6$. Substituting this expression into Eq. A3, and hereafter taking $b_{1}=b_{2}=b$, we find:

$$
\sqrt{\bar{N}_{1}} \frac{\delta R_{g, 1}^{2}}{R_{g 0,1}^{2}}=\frac{F(\alpha)}{\alpha}-2 F(0) \cdot \chi_{e} N_{1}
$$

where

$$
F(\alpha) \equiv \frac{6^{3 / 2}}{4 \pi^{2}} \int^{*} d y \frac{f\left(y^{2}\right) y^{2}}{g\left(\alpha y^{2}\right)}
$$

where $\alpha \equiv N_{2} / N_{1}$, and where $y \equiv q^{2} R_{g 0,1}^{2} . F(\alpha)$ is convergent when $\alpha=0$ and is UV-divergent for any nonvanishing $\alpha$. In the latter case, a constant $4 / 3$ has to be subtracted from the integrand to account for the renormalization of the statistical segment length.

Three special cases of this result are noted in the main text: The fractional shrinkage of $R_{g}^{2}$ in a homopolymer melt, which is discussed in subsection IVA is obtained by setting $\chi_{e}=0$ and $\alpha=1$. This yields $\sqrt{\bar{N}} \delta R_{g}^{2} / R_{g, 0}=$ $F(1)=-1.42$. In section IVB the curve shown in Fig. 5 is given by $F(\alpha) / \alpha$. Its asymptotic behavior in the limit of a small molecule solvent, $\alpha \rightarrow 0$, is given by $F(0) / \alpha=0.31 / \alpha$. In section IVC the $\chi_{e} N$ dependence of the fractional change of $R_{g}^{2}$ in the limit of $\phi_{1} \rightarrow 0$ is given by $\partial\left(\sqrt{\bar{N}} d R_{g}^{2} / R_{g, 0}^{2}\right) / \partial\left(\chi_{e} N\right)=-2 F(0)=-0.62$.

\section{APPENDIX B: MEAN-SQUARED END-TO-END DISTANCE}

We calculate the one-loop correction to the meansquared end-to-end distance $R^{2}$ using the method described in $^{32}$ (appendix III of chapter 5). There, the same quantity was calculated for a finite segment of an infinitely long chain in a semi-dilute solution, using a Lorentzian approximation for the screened interaction. The analogous result for a chain of species 1 in an incompressible binary blend is obtained by using the appropriate screened interaction $G_{i j}$. The final result for a symmetric blend $\left(N_{1}=N_{2}\right.$ and $\left.b_{1}=b_{2}\right)$ is

$$
\frac{\delta R_{1}^{2}}{R_{0}^{2}}=\frac{\sqrt{24}}{\pi^{2} \sqrt{\bar{N}}} \int^{*} d y \frac{h\left(y^{2}\right) y^{4}}{g\left(y^{2}\right)} \frac{1-2 \phi_{2} \chi_{e} N g\left(y^{2}\right)}{1-2 \phi_{1} \phi_{2} \chi_{e} N g\left(y^{2}\right)} \text { (B1) }
$$

where $y \equiv q^{2} R_{g 0}^{2}$, and

$$
h(x) \equiv\left(\frac{1}{x^{2}}+\frac{4}{x^{3}}+\frac{6}{x^{4}}\right) \mathrm{e}^{-x}+\frac{2}{x^{3}}-\frac{6}{x^{4}} .
$$

This expression reduces to Eq. (15) of Ref ${ }^{12}$ for a onecomponent melt $\left(\phi_{2}=0\right)$.

The integral is divergent in the high- $q$ region, and the dangerous term in the integrand is " 1 ". This is precisely the divergent term identified by Edwards, which he identified with as a renormalization of the statistical segment length ${ }^{32}$. We subtract this term from the integrand in order to obtain a UV convergent prediction for $\delta R^{2}$, which is the deviation of $R^{2}$ from the value $R_{0}^{2}(N)$ predicted by a Gaussian model with a statistical segment length equal to that obtained for infinitely long chains. For a pure homopolymer melt, by numerically completing the convergent integral, we recovered the result reported in ${ }^{12}: \delta R^{2} / R_{0}^{2}=$ $-1.40 / \sqrt{\bar{N}}$. 
1. Schweizer, K.; Curro, J. Advances in Polymer Science 1994, 116, 321-377.

2. Lipson, J. E. G. Macromol. Theory Simul. 1998, 7, 263-282.

3. de la Cruz, M. O.; Edwards, S. F.; Sanchez, I. C. J. Chem. Phys. 1988, 89, 1704-1708.

4. Bates, F. S.; Fredrickson, G. H. Macromolecules 1994, 27, 1065-1067.

5. Fredrickson, G. H.; Liu, A. J.; Bates, F. S. Macromolecules 1994, 27, 2503-2511.

6. Holyst, R.; Vilgis, A. J. Chem. Phys. 1993, 99, 4835-4844.

7. Holyst, R.; Vilgis, A. Phys. Rev. E 1994, 50, 2087-2092.

8. Wang, Z.-G. Macromolecules 1995, 28, 570-576.

9. Wang, Z.-G. J. Chem. Phys. 2002, 117, 481-500.

10. Grzywacz, P.; Qin, J.; Morse, D. C. Phys. Rev. E 2007, 76, 061802.

11. Beckrich, P.; Johner, A.; Semenov, A. N.; Obukhov, S. P.; Benoît, H.; Wittmer, J. P. Macromolecules 2007, 40, 38053814.

12. Wittmer, J. P.; Beckrich, P.; Meyer, H.; Cavallo, A.; Johner, A.; Baschnagel, J. Phys. Rev. E 2007, 76, 011803.

13. des Cloizeaux, J.; Janink, G. Polymer in Solution: Their Modelling and Structure; Oxford: Oxford, U.K., 1990.

14. Ohta, T.; Nakanishi, A. J. Phys. A: Math. Gen. 1983, 16, 4155-4170.

15. Nakanishi, A.; Ohta, T. J. Phys. A: Math. Gen. 1985, 18, 127-139.

16. Fetters, L. J.; Lohse, D. J.; Richter, D.; Witten, T. A.; Zirkel, A. Macromolecules 1994, 27, 4639-6447.

17. de Gennes, P. G. J. de Phys. 1977, 38, L441-443.

18. Morse, D. C. Annals of Physics 2006, 321, 2318.
19. Barrat, J.-L.; Fredrickson, G. H. J. Chem. Phys. 1991, 95, 1281-1289.

20. Semenov, A. N.; Obukhov, S. P. J. Phys: Condens. Matter 2005, 17, S1747-S1775.

21. Wittmer, J. P.; Beckrich, P.; Johner, A.; Semenov, A. N.; Obukhov, S. P.; Meyer, H.; Baschnagel, J. Eur. Phys. Lett. 2007, 77, 56003.

22. Yamakawa, H. Modern Theory of Polymer Solutions; Harper \& Row: New York, 1972.

23. de Gennes, P.-G. Scaling Concepts in Polymer Physics; Cornell University Press: , 1979.

24. Mueller, M.; Binder, K. Macromolecules 1995, 28, 18251834.

25. Mueller, M.; de Pablo, J. J. Lect. Notes Phys. 2006, 703, 67126.

26. Deutsch, H. P.; Binder, K. J. Phys. II France 1993, 3, 10491073.

27. Shirvanyants, D.; Panyukov, S.; Liao, Q.; Rubinstein, M. Macromolecules 2008, 41, 1475-1485.

28. Mueller, M. Macromolecules 1998, 31, 9044-9057.

29. McQuarrie, D. Statistical Mechanics; Harper Collins Publishers: New York, 1976.

30. Schweizer, K. S.; Curro, J. G. Chemical Physics 1990, 149, 105-127.

31. Aksimentiev, A.; Holyst, R. Macromol. Theory Simul. 1998, 7, 447-456.

32. Doi, M.; Edwards, S. F. The Theory of Polymer Dynamics; Oxford University Press: Oxford Science Publications, 1986. 